\title{
The Interaction of High-Speed Turbulence with Flames: Global Properties and Internal Flame Structure
}

\author{
A.Y. Poludnenko*, E.S. Oran \\ Laboratory for Computational Physics and Fluid Dynamics, Naval Research Laboratory, Washington, DC 20375, USA
}

\begin{abstract}
We study the dynamics and properties of a turbulent flame, formed in the presence of subsonic, high-speed, homogeneous, isotropic Kolmogorov-type turbulence in an unconfined system. Direct numerical simulations are performed with Athena-RFX, a massively parallel, fully compressible, high-order, dimensionally unsplit, reactive-flow code. A simplified reaction-diffusion model represents a stoichiometric $\mathrm{H}_{2}$-air mixture. The system being modeled represents turbulent combustion with the Damköhler number $D a=0.05$ and with the turbulent velocity at the energy injection scale 30 times larger than the laminar flame speed. The simulations show that flame interaction with high-speed turbulence forms a steadily propagating turbulent flame with a flame brush width approximately twice the energy injection scale and a speed four times the laminar flame speed. A method for reconstructing the internal flame structure is described and used to show that the turbulent flame consists of tightly folded flamelets. The reaction zone structure of these is virtually identical to that of the planar laminar flame, while the preheat zone is broadened by approximately a factor of two. Consequently, the system evolution represents turbulent combustion in the thin-reaction zone regime. The turbulent cascade fails to penetrate the internal flame structure, and thus the action of small-scale turbulence is suppressed throughout most of the flame. Finally, our results suggest that for stoichiometric $\mathrm{H}_{2}$-air mixtures, any substantial flame broadening by the action of turbulence cannot be expected in all subsonic regimes.
\end{abstract}

Keywords: Turbulent premixed combustion, Turbulence, Flamelet, Flame structure, Hydrogen

\section{Introduction}

In the last twenty years, studies of premixed turbulent combustion have witnessed an explosive growth. The motivation for this comes from a remarkably broad range of both engineering and basic science applications - from the design of internal combustion engines, to problems of ensuring safety in mines, to the dynamics and properties of turbulent thermonuclear flames powering type Ia supernovae. Such rapid development has been enabled by significant advances in experimental techniques, in particular in terms of the diagnostics, as well as by the substantial increase in the capabilities of the computational infrastructure and numerical algorithms, all of which made direct numerical simulation (DNS) a viable, and often indispensable, tool for turbulent combustion research.

Despite the significant increase in the body of experimental and numerical data, the overall paradigm still prevailing today is reflected in a variety of combustion regime diagrams (e.g., see [1, 2, 3] and references therein), which attempt to provide a comprehensive classification of the different modes of turbulence-flame interaction. The key underlying assumption is that such classification can be made based on a very limited set of parameters, namely, the characteristic large-scale turbulent velocity and the spatial scale. With this ansatz, the diagrams can be constructed by comparing various turbulent timescales, e.g., eddy turnover timescales on the integral and Kolmogorov scales, with those of the unperturbed laminar flame. The resulting classifications include such regimes as "wrinkled" and "corrugated flamelets," as well as "thin," "broken," and "distributed reaction zones," which suggest the typical qualitative structure of the turbulent flame under specific conditions.

${ }^{*}$ Corresponding author. Fax: +1 2027674798 .

Email address: apol@lcp.nrl.navy.mil (A.Y. Poludnenko) 
The basis for this paradigm is the picture which was first suggested by Damköhler almost 70 years ago [4, 5, 6]. Large-scale motions are responsible for the overall stretching and folding of the flame that constitutes the flame brush. This process increases the flame surface area, which directly determines the global properties of the turbulent flame, such as its width and speed. Therefore, depending on whether the characteristic timescale of these large-scale motions is greater or smaller than the laminar flame self-crossing time, the flame is either able or not to reorganize and adjust itself to the action of turbulence.

On the other hand, small scales penetrate inside individual flamelets, thus affecting their internal structure and broadening their preheat and reaction zones. The efficiency of this process is controlled by the magnitude of the Kolmogorov scale with respect to the laminar flame width, $\delta_{L}$, or the size of the reaction zone in the laminar flame. Once the Kolmogorov scale becomes smaller, it is hypothesized that the turbulent cascade is able to penetrate the internal structure of the flamelets. As a result, turbulent transport becomes comparable to, or exceeds, molecular diffusive processes, and the flamelet width and burning velocity increase.

Overall, in this picture the actions of large and small scales are quite distinct. Large scales determine the global turbulent burning speed, which increases compared to the laminar burning speed in proportion to the increase in the flamelet surface area. At the same time, small scales affect the turbulent flame speed only by modifying the local burning velocity of individual flamelets.

This dichotomy in the action of the large and small scales by and large has its root in the behavior of a passive scalar in turbulent nonreactive flows. The fact that in nonreactive turbulence the inertial range extends to scales smaller than $\delta_{L}$, however, does not automatically mean that turbulent motions of the same intensity penetrate inside the flamelets, nor does it mean that they have the same effect on the internal flamelet structure as they would have on a passively advected scalar.

To date, the majority of research efforts have focused on regimes characterized by smaller ratios of turbulent velocities to the laminar flame speeds (see reviews by [5, 6]), where there are the most applications of practical interest. In these cases, penetration by small-scale turbulent motions into the internal flamelet structure is either completely suppressed, or it is heavily dominated by flame wrinkling due to large-scale motions. Results of these efforts are in agreement with the Damköhler concept discussed above, and they support the classification of such regimes as "wrinkled" or "corrugated" flamelets.

The investigation of the regimes in which turbulent velocities are significantly larger than the laminar flame speed, $S_{L}$, on all scales, including that of the laminar flame thickness, presents a number of both experimental and numerical challenges. Hereafter, we refer to this mode as high-speed turbulent combustion, which encompasses regimes such as thin and broken reaction zones, as well as distributed flames. These are the regimes in which substantial flame broadening by turbulent transport has been hypothesized. We also assume that turbulence is the only process that can affect the structure and properties of the flame. We do not consider situations in which the flame is altered or broadened by fuel preconditioning, compression of the overall system, or propagation of large-scale shocks.

Probing such regimes experimentally requires either high turbulent intensities or low laminar flame speeds. Generating and sustaining high-speed reactive turbulence with desired properties may be difficult in a laboratory setting. More important, however, is that if $S_{L}$ is high, the maximum ratios of the integral turbulent velocity $U_{l}$ to $S_{L}$ cannot be too large, or the overall flow will no longer be subsonic. Therefore, almost all studies that were able to achieve $U_{l} / S_{L} \gtrsim 10-20$ used lean hydrocarbon fuels, which have low values of $S_{L}[7,8$, , 9] (also see the review by [5]).

Of particular interest is the work by Dunn et al. [10], in which a piloted premixed jet burner was used to achieve $U_{l} / S_{L}$ in the range $40-390$, corresponding to Karlovitz numbers $K a=100-3500$ and Damköhler numbers $D a=$ $0.069-0.0053$. In their lowest jet-velocity case, which would be characterized as being well into the distributed-flame regime, there was no evidence of flame broadening based on the temperature and $\mathrm{OH}$ images. This is in contradiction to predictions of the traditional classification of the combustion regimes. Substantially higher turbulent intensities were required to produce a flow structure that was characterized by the authors as a distributed mode of burning, although this determination was done mostly on qualitative grounds.

In numerical simulations, generating turbulence of arbitrary intensity does not present any complications. Instead, modeling high-speed turbulent reactive flows faces different challenges that can be as restrictive as those encountered in experiments. One major difficulty is that the Kolmogorov scale can be substantially smaller than $\delta_{L}$, thereby making fully-resolved DNS difficult even with the largest computational resources. Furthermore, when fluid velocities reach a substantial fraction of the speed of sound, it is necessary to use fully compressible integration algorithms for which the numerical time step is limited by the CFL condition. This further increases the computational cost. 
Several studies of chemical flames achieved values of $U_{l} / S_{L}$ in the range $3-10$ in a flat-flame configuration [11, 12, 13] and $U_{l} / S_{L}=20$ in the case of a bunsen flame [14] (also see the review by [5]). In all of these studies, the reaction zone has a thin sheet-like structure. Moreover, Bell et al. [12] demonstrate that the increase in turbulent velocity can almost completely be accounted for by the corresponding increase in the flamelet surface area, although the former appears to be $\sim 10 \%$ larger than the latter. Note that the studies considering the flat-flame configuration $[11,12,13]$ used decaying turbulence, which precluded the system from reaching a steady state.

Aspden et al. [15] reported flame broadening and formation of a distributed reaction zone in a DNS under the action of fast turbulence. This study considers the interaction of a thermonuclear flame, driven by ${ }^{12} \mathrm{C}-{ }^{12} \mathrm{C}$ reactions, with steady driven turbulence under the conditions characteristic of the interior of a white dwarf during the late stages of the type Ia supernova explosion. In the fastest turbulence case, the value of $U_{l} / S_{L} \approx 70$ was achieved corresponding to the Karlovitz number $K a=228$, where $K a=\left(\delta_{L} / L_{G}\right)^{1 / 2}$ and $L_{G}$ is the Gibson scale defined in eq. (11). This simulation showed the formation of a broad turbulent flame with a complex temperature and burning-rate structure. Moreover, joint PDFs of various quantities, such as carbon mass fraction and temperature, were characteristic of a Lewis number $L e=1$ system, whereas the actual values of $L e$ are extremely large. This fact, along with wide smooth averaged profiles of various quantities throughout the turbulent flame, led to the conclusion that the resulting flame is dominated by turbulent transport and presents an example of a broad distributed reaction zone.

Even given the efforts discussed above, the physics of high-speed turbulent combustion still has many unanswered questions. With the exception of the experimental results of [10] and numerical results of [15], evidence of significant flame broadening and the formation of distributed reaction zones is scarce [5]. Moreover, results of all studies suggest that such broadening, even if it does occur, requires substantially higher turbulent intensities than traditionally predicted. It remains unclear whether the effects of turbulence are indeed so much weaker than can be expected based on simple physical grounds and, if this is the case, what mechanisms are responsible for suppressing the action of turbulence.

The work by [10] and [15] emphasizes the importance of detailed and reliable diagnostics. While both studies present interesting experimental and numerical investigations of the previously unexplored regimes, they both rely on indirect evidence in their determination of the observed flame broadening. Of paramount importance are the measurements that would, for example, directly answer questions regarding the actual multidimensional structure of the region in which most of the energy release takes place, the nature of flame broadening, and the effects of turbulence on different regions of the flame.

Our goal in this work is to address several key questions concerning the dynamics and properties of the turbulent flames formed under the action of high-speed turbulence. First of all, it is important to understand (1) the global properties of the turbulent flame brush in this regime, i.e., its width, speed, etc.; (2) the internal structure of the flame brush; and (3) the internal structure of the flamelets folded inside the flame brush, if such flamelets can be identified at all. It is important to address the last two points quantitatively through direct measurement. All of these characteristics are determined by turbulence-flame interactions, and the resulting system is the product of the collective action of the full turbulent energy cascade. Therefore, it is also important to determine the relative role of large- and small-scale motions on the global and local properties of the turbulent flame.

In this paper, we begin to address these questions by presenting three simulations, which are designed to represent the turbulent combustion process in an unconfined (ideally infinite) space. Initially, the flame is a planar surface separating half of the domain containing fuel from the half of the domain containing products and immersed in a high-intensity turbulent flow field with a Kolmogorov-type spectrum [16]. It is assumed that the energy injection scale, as well as the turbulent integral scale, are both finite and much smaller than the overall system size. The evolution of such a system represents the evolution of an infinite globally planar turbulent flame brush. Such an idealized setting allows us to exclude effects such as walls, boundaries, and the system geometry from consideration and to focus on the role played by turbulence.

We consider driven turbulence of sufficiently high intensity to place the system into the regime which is transitional, according to the traditional classifications, between thin and broken reaction zones. This is the regime in which turbulence would be expected to have an effect on the structure not only of the preheat but also of the reaction zone. By considering a stoichiometric reactive mixture, we minimize the thermo-diffusive effects as well as the possibility of flame extinction under the action of intense turbulence. We use a simplified reaction model designed to accurately represent the laminar flame properties of the stoichiometric $\mathrm{H}_{2}$-air mixture. A relatively high laminar flame speed of this fuel leads to turbulent velocities that are a substantial fraction of the sound speed in cold fuel. The resulting 
flow can no longer be considered incompressible, which requires the use of a high-order, dimensionally unsplit, fully compressible integration method.

The three simulations on which we focus in this paper are part of a larger series of numerical models. Their goal is to survey the full range of subsonic high-speed turbulent combustion regimes in a variety of reactive mixtures. This work is intended to identify the overall framework for future analysis of such numerical models.

\section{Formulation of the Problem}

\subsection{Physical model}

We solve the system of unsteady, compressible, reactive flow equations,

$$
\begin{aligned}
\frac{\partial \rho}{\partial t}+\nabla \cdot(\rho \mathbf{u}) & =0, \\
\frac{\partial \rho \mathbf{u}}{\partial t}+\nabla \cdot(\rho \mathbf{u} \otimes \mathbf{u})+\nabla P & =0, \\
\frac{\partial E}{\partial t}+\nabla \cdot((E+P) \mathbf{u})-\nabla \cdot(K \nabla T) & =-\rho q \dot{w}, \\
\frac{\partial \rho Y}{\partial t}+\nabla \cdot(\rho Y \mathbf{u})-\nabla \cdot(\rho D \nabla Y) & =\rho \dot{w} .
\end{aligned}
$$

Here $\rho$ is the mass density, $\mathbf{u}$ is the velocity, $E$ is the energy density, $P$ is the pressure, $Y$ is the mass fraction of the reactant, $K$ and $D$ are the coefficients of thermal conduction and molecular diffusion, $q$ is the chemical energy release, and $\dot{w}$ is the reaction source term. The equation of state is that of an ideal gas. We do not include physical viscosity in our model. Therefore, dissipation is provided by the numerical viscosity. This issue, and the way we use the inherent numerical viscosity in the algorithm, will be discussed in detail in $\S 2.4$. Finally, since our method of turbulence driving, described in $\$ 2.2$, does not involve modifying the fluid equations, eqs. (1) - (4) do not contain any forcing term.

Chemical reactions are modeled using the first-order Arrhenius kinetics

$$
\frac{d Y}{d t} \equiv \dot{w}=-A \rho Y \exp \left(-\frac{Q}{R T}\right)
$$

where $A$ is the pre-exponential factor, $Q$ is the activation energy, and $R$ is the universal gas constant.

We assume that both thermal conduction and species diffusion have similar temperature dependence

$$
D=D_{0} \frac{T^{n}}{\rho}, \quad \frac{K}{\rho C_{p}}=\kappa_{0} \frac{T^{n}}{\rho},
$$

where $D_{0}, \kappa_{0}$, and $n$ are constants, and $C_{p}=\gamma R / M(\gamma-1)$ is the specific heat at constant pressure. The Lewis number $L e=\kappa_{0} / D_{0}$ is set to be equal to one and it is independent of the thermodynamic conditions of the flow. External forces, the Soret and Dufour effects, pressure gradient diffusion as well as the effects of radiation are assumed to be negligible. The last assumption, however, requires further verification. Contributions of the molecular dissociation, atomic ionization, and radiative heat losses to the equation of state are assumed to be absorbed by the model value of the adiabatic index $\gamma$.

Reaction model parameters are summarized in Table 1] They are based on the simplified reaction model of [17] designed to represent the stoichiometric $\mathrm{H}_{2}$-air mixture. The values of the transport coefficients are comparable to those of air at the same conditions. This reaction model reproduces the key characteristics both of the laminar flames and the multidimensional detonations in the given reactant mixture, such as the laminar flame width, speed, detonation velocity and detonation cell size, etc. as well as the dependence of these quantities on pressure and temperature. It has also been demonstrated to provide good qualitative agreement with experimental data in more complex applications involving flame acceleration and deflagration-to-detonation transition in channels with obstacles [17, 18]. These properties of the reaction model, along with its low computational cost, make it a practical choice for large-scale 
multidimensional simulations aimed at qualitative analysis of the turbulence-flame interaction in stoichiometric $\mathrm{H}_{2}$ air mixtures and other similar fuels. It is important to keep in mind that the first-order Arrhenius kinetics is not able to capture the full complexity of hydrogen combustion. Therefore, certain care must be exercised when comparing results obtained using this model with the actual experimental data.

\subsection{Numerical method}

To model the process of turbulence-flame interaction, we use the code Athena-RFX [19] - the reactive flow extension of the magnetohydrodynamic code Athena [20, 21, 22]. Athena-RFX is a fixed-grid massively parallel code. It implements several higher-order fully conservative Godunov-type methods for integration of fluid equations. In this work we use the method based on the fully unsplit corner transport upwind (CTU) algorithm of [23] and its three-dimensional (3D) extension presented in [24]. In particular, Athena implements a variant of this method [21], which requires six Riemann solutions per cell instead of the twelve in the original method of [24]. This integration scheme uses PPM spatial reconstruction [25] in conjunction with the approximate nonlinear HLLC Riemann solver to achieve 3rd-order accuracy in space and 2nd-order accuracy in time. A detailed description and an extensive suite of tests of the hydrodynamic integration algorithm and its implementation in Athena can be found in [20, 21].

Diffusive transport is incorporated into the hydrodynamic integration algorithm by calculating net diffusive fluxes across each cell along each dimension. This is done using second-order finite differences with flux matching to ensure that the conservation properties of the method are preserved. Those fluxes are then added to the hydrodynamic fluxes prior to the final update of the state vector in each cell. Source terms, describing chemical reactions, are coupled using Strang splitting to ensure the second-order accuracy of the overall solution.

The overall resulting solver is formally second-order accurate and it is capable of providing the accuracy of the planar laminar flame solution of $\lesssim 1-2 \%$ even with the resolution of $\sim 4$ cells per $\delta_{L}$. We refer to [19] for further details of the implementation of the reactive-diffusive extensions in Athena-RFX, as well as the results of tests including convergence studies.

\subsection{Turbulence driving method}

We investigate the process of flame interaction with steady homogeneous isotropic turbulence, described by the classical Kolmogorov theory [16]. In the absence of persistent energy injection into the system at a large scale, turbulence decays on a characteristic time scale of the large-scale eddy turnover time. Under conditions considered later in this paper, this time scale is almost four times shorter than the laminar flame self-crossing time $\delta_{L} / S_{L}$ (see Tables 1 and 3. Consequently, in order to study the quasi-steady regime of turbulence-flame interaction, the flow must be constantly stirred at the largest scale to create and maintain a steady energy cascade to smaller scales.

We use a spectral turbulence-driving method similar to the one used in [26, 27]. For completeness here we summarize the key algorithmic stages of the method. More detailed discussion of the method, along with the analysis of its properties and an extensive suite of tests demonstrating its performance, can be found in [19].

As the first step, we consider Fourier transforms of the velocity perturbations $\delta \hat{\mathbf{u}}^{\prime}(\mathbf{k})$, with each component $\delta \hat{u}_{i}^{\prime}$ being an independent realization of a Gaussian random field with zero mean and unit variance. Then a given isotropic energy injection spectrum $\delta \mathcal{E}(k)$ is superimposed on $\delta \hat{\mathbf{u}}^{\prime}(\mathbf{k})$

$$
\delta \hat{u}_{i}(k)=\frac{\sqrt{\delta \mathcal{E}(k)}}{k} \delta \hat{u}_{i}^{\prime}(k),
$$

where $k=\|\mathbf{k}\|$. In principle, the amplitude and phase of each component $\delta \hat{u}_{i}$ at every point $\mathbf{k}$ can be independently adjusted to produce an energy-injection pattern of arbitrary complexity.

The computational domain has volume $V=L_{1} L_{2} L_{3}$, where $L_{1} \leq L_{2} \leq L_{3}$. In this work we inject energy only at the scale $L=L_{1}$ to obtain the Kolmogorov-type spectrum. In particular,

$$
\delta \mathcal{E}=\left\{\begin{array}{l}
0 \forall \mathbf{k}=\left\{k_{1}, k_{2}, k_{3}\right\}: k_{1}=k_{2}=k_{3}=0, \quad k_{i} \neq \frac{2 \pi}{L_{1}}, \\
1 \forall \mathbf{k}=\left\{k_{1}, k_{2}, k_{3}\right\}: k_{i}=0, \quad k_{i}=\frac{2 \pi}{L_{1}} .
\end{array}\right.
$$

The mode with all zero components is not driven since that would simply be inducing a uniform bulk flow through the domain. 
Finally, non-solenoidal components of the velocity perturbations are removed using the orthogonal projection operator, which ensures that $\nabla \cdot \delta \mathbf{u}(\mathbf{x})=0$.

An inverse Fourier transform of $\delta \hat{\mathbf{u}}(\mathbf{k})$ gives $\delta \mathbf{u}(\mathbf{x})$, the velocity perturbation field in the physical space. Then $\delta \mathbf{u}(\mathbf{x})$ are added to the velocity field $\mathbf{u}(\mathbf{x})$ in the domain on every time step, after first being normalized to ensure constant rate $\varepsilon$ of kinetic-energy injection. Moreover, the total momentum in the perturbation field is subtracted from $\delta \mathbf{u}(\mathbf{x})$ to ensure that no net momentum is added to the domain, i.e., $\int \rho \delta \mathbf{u}=0$. The overall perturbation pattern is regenerated at every time interval $\Delta t_{v p} \approx 5 \Delta x / c_{s}$, where $c_{s}$ is the sound speed in the domain.

This turbulence driving method produces statistically steady, isotropic, and homogeneous turbulent flows with energy spectra of arbitrary complexity [19]. In particular, it is possible to obtain Kolmogorov-type turbulence with the inertial range of the energy cascade extending all the way to the energy injection scale. This is crucial, given the limited range of spatial scales typically accessible in a simulation. The method does not introduce any large-scale anisotropies or global flows. Moreover, since the velocity perturbation field is purely solenoidal, no compressions or rarefactions are artificially induced as a result of driving. This is particularly important in the case of the reactive flows, in which the rate of energy generation can be very sensitive to the local variations in temperature and pressure.

\subsection{Problem setup and summary of performed simulations}

Table 2 summarizes key parameters of the performed simulations. The main difference between the three models is the resolution, which progressively increases from $\Delta x=\delta_{L} / 8$ in $\mathrm{S} 1$ to $\Delta x=\delta_{L} / 32$ in S3. One of the principal goals of this work, discussed in $\S 1$ is to investigate the effects of small-scale motions and, thereby, to differentiate them from the role played by large scales. The most direct way to achieve this is by varying the viscosity. In particular, changing the resolution changes the numerical dissipation, and this causes the effective viscosity in the domain to vary. This allows us to control the amount of kinetic energy contained on scales comparable to or smaller than $\delta_{L}$, while preserving the energy spectrum on larger scales.

Figure 11 shows instantaneous spectra in the domain prior to ignition in simulations S1 - S3. These spectra represent the flow field in the nonreactive turbulence. While the spectra are virtually the same on scales $\gtrsim 2 \delta_{L}$, they vary greatly in energy contained on small scales. In the least resolved case, $\mathrm{S} 1$, motions on all scales $\lesssim \delta_{L}$ are affected by dissipation. In the most resolved case, S3, the inertial range extends to scales which, in the reactive flow, would be deep inside the flame. As a result, energy on the small scales varies by up to a factor of 30 between these two cases. By comparing the flow structure in these simulations, it is then possible to establish the effects of small-scale turbulence on the turbulent flame. In particular, this allows us to determine the degree to which turbulent cascade is able to penetrate and alter the internal structure of the flame.

A similar analysis could be performed by including physical viscosity in the model and by varying it parametrically. Such an analysis, however, would not represent the behavior of an actual fuel any more than the one which relies on numerical viscosity, unless the viscosity parameter is close to its real physical value. Moreover, there are several practical complications associated with using physical viscosity instead of the numerical one. Figure 1 shows the wavenumbers associated with the physical Taylor and Kolmogorov scales in both the product and fuel in a stoichiometric $\mathrm{H}_{2}$-air mixture. These values were determined using the temperature dependence of the viscosity coefficient similar to that of other diffusion coefficients, namely $v=v_{0} T^{n} / \rho$ where $n=0.7$ and $v_{0}=2.9 \times 10^{-5} \mathrm{~g} / \mathrm{s} \cdot \mathrm{cm} \cdot \mathrm{K}^{0.7}$ corresponding to $\operatorname{Pr}=S c=1$ [17]. It can be seen that resolving the Kolmogorov scale in the cold fuel would require substantially higher grid resolutions. Achieving such resolution, while maintaining a reasonably large separation between the scales $L$ and $\delta_{L}$, would be difficult to impossible with current computational resources. For higher turbulent intensities, $\eta_{F}$ would be even smaller. In addition, physical viscosity results in a much shallower and wider dissipation range. Given the limited range of spatial scales accessible in a numerical simulation, this would substantially restrict the possible extent of the inertial range in the flow.

Figure 1 shows that in cases S1 and S3, the numerical Taylor scales, i.e., the largest scales in the domain affected by dissipation, are similar to physical Taylor scales in product and fuel, respectively. Therefore, the performed simulations do capture both the smallest and the largest extents of the inertial range present in the physical stoichiometric $\mathrm{H}_{2}$-air mixture.

These considerations show that fully resolved DNS models with physical viscosity are impractical for our study. More important, however, is that it is possible to perform a detailed analysis of the effects of small scales on the turbulent flame by varying numerical resolution without resorting to fully resolved DNS simulations. In particular, 
if a certain property of the turbulent flame does not change or appears to be converged with increasing resolution, it indicates that its evolution is determined only by large scales. On average, the flow on large scales is independent of small scales. Therefore, if large-scale motions are properly captured in a simulation, as is the case in the models presented here, then the evolution of such a property cannot be affected by the even smaller scales that would exist in a real system due to a much smaller physical viscosity.

Methods that rely on numerical viscosity to provide kinetic-energy dissipation in the grid, while explicitly capturing the inertial range of the energy cascade, are often referred to as implicit large-eddy simulations (ILES). The feasibility of this approach was first suggested by Boris [28] and it has since been extensively used in turbulence modeling. In particular, a detailed analysis of the effects of numerical viscosity in the context of the PPM method, similar to the one used in this work, was performed by Porter et al. [29]. An example of similar analysis for a different higher-order algorithm, namely flux-corrected transport, can be found in [30]. A comprehensive overview of this class of methods is given in [31].

Aside from controlling the amount of energy on small scales, grid resolution also plays its traditional role. It must be high enough to provide sufficient accuracy of the overall solution on all scales. As was demonstrated in [19] for Athena-RFX, a resolution of even 8 cells per $\delta_{L}$ is sufficient to reproduce very accurately properties of the planar laminar flame. A priori, it is not clear, however, that this resolution is enough to capture the complex dynamics of the turbulent flame, which contains highly distorted flamelets. Strong turbulence can fold individual flame sheets with the curvature radius comparable to $\delta_{L}$ or bring two flame sheets together. This would form cusps, whose velocity of propagation can be substantially larger than that of a planar laminar flame. Properly capturing the rate of burning in such cusps depends sensitively on the code's ability to accurately model thermal flux focusing in regions of high flame curvature. Even the high-order dimensionally unsplit algorithms introduce some degree of anisotropy to the thermal flux, which tends to align itself with the grid. At low resolution, this can substantially affect thermal transport in cusps. The increasing resolution in simulations $\mathrm{S} 1-\mathrm{S} 3$ allows us to carry out a convergence study to address these issues.

All simulations were performed in the domain with the high length-to-width ratio of 16:1. The longest dimension of the domain is assumed to be along the $z$-axis. Such long domains are computationally expensive, however they are needed to be able to follow the flame evolution for extended periods of time, namely for $16 \tau_{\text {ed }}$. We learned empirically that this extended domain size is necessary to accommodate flame-brush motion with respect to the grid during that time and to ensure that the flame brush remains sufficiently far from the outflow boundaries in order to minimize their effect on the system.

The domain in all calculations was initialized with uniform density $\rho_{0}$ and temperature $T_{0}$ (see Table 1). In simulations $\mathrm{S} 1$ and $\mathrm{S} 2$, initial fluid velocities were set to 0 . In contrast, in S3 the velocity field was initialized with the ideal energy spectrum $\mathcal{E}(k) \propto k^{-5 / 3}$ extending from the energy injection scale $L$ to the numerical Kolmogorov scale $\eta=2 \Delta x$. This initial spectrum was normalized to ensure that at $t=0$ the total kinetic energy in the domain was equal to its predicted steady-state value as described in [19]. The main advantage of this method of velocity initialization is that it allows the flow to reach its equilibrium state much faster. In particular, the equilibration time is $2 \tau_{e q}$ in $\mathrm{S} 3$, whereas it is $3 \tau_{e q}$ in S1 and S2 in which initial velocities are zero [19]. This is due to the much shorter time needed to populate all spectral modes and for the steady energy cascade to develop. Moreover, the rate of energy dissipation is close to its equilibrium value practically from $t=0$, thus preventing the build-up of excess kinetic energy and, thereby, also shortening the equilibration phase. A detailed discussion of this method of turbulent flow field initialization can be found in [19].

It is shown in [19] that in nonreactive turbulence, the long-term system evolution is not sensitive to the form of the velocity field in the domain at $t=0$, provided that sufficient time was given for the system to reach its equilibrium state. Further results will demonstrate that this is also the case in reactive turbulence.

Steady driving with constant rate $\varepsilon$ of energy injection into the domain was applied to the system for the total duration of all simulations. The energy injection scale is always assumed to be the domain width $L$. The value of $\varepsilon$ was chosen to produce a turbulence field of sufficiently high intensity that, however, was low enough to minimize the probability of creating the weak transonic shocklets that arise from intermittency in turbulent flow. In fast turbulent flows, which can still be nominally characterized as subsonic based, for instance, on the value of $U_{r m s}$, such shocklets can represent a substantial part of the flow field [19].

Table 2 lists a number of velocity characteristics along with the integral scale of the steady-state turbulent flow prior to the moment of ignition. In the simulations, we do not follow the evolution of the nonreactive turbulence in 
the steady state long enough to extract time-averaged values of those parameters. Therefore, Table[2]lists theoretically predicted equilibrium values based on the expressions given in [19]. Analysis performed in [19] shows that such estimates generally are accurate to a few percent. All derived quantities listed in Table 2, such as $\tau_{e d}, L_{G}, D a, M a_{F}$, and $M a_{P}$, are also based on those values.

In our model we do not include any artificial cooling mechanisms to compensate for the turbulent heating of the flow. Based on the analysis given in [19], the amount of energy injected into the domain within one large-scale eddy turnover time $\tau_{e d}$ is $\approx 80 \%$ of the total steady-state kinetic energy in the nonreactive turbulent flow. The typical time step is $\sim 10^{-9}-10^{-8} \mathrm{~s}$, or $\sim 10^{-4} \tau_{e d}$ (see Table 2). Therefore, the amount of energy injected into the domain on every time step is $\lesssim 10^{-4}$ of the equilibrium kinetic energy. The corresponding relative increase in the internal energy and temperature due to the turbulent heating in the nonreactive flow within $\tau_{e d}$ is [19]

$$
\frac{E_{t}-E_{t, 0}}{E_{t, 0}}=\frac{T-T_{0}}{T_{0}}=\frac{\varepsilon \rho_{0} \tau_{e d}}{E_{t, 0}}=D_{K} \gamma(\gamma-1) M a_{F}^{2},
$$

where the constant $D_{K}=0.5$ and $E_{t, 0}$ is the thermal energy in the domain at $t=0$. Using values of $\gamma$ from Table 1 and $M a_{F}$ from Table 2, this increase is $\approx 0.6 \%$ of their initial values or, equivalently, $\approx 1.8 \mathrm{~K}$ over $\tau_{e d}$. Such a small amount of heating cannot result in any significant fuel preconditioning and, thus, it does not affect any of the conclusions.

In simulations S1 and S2, the flow field was allowed to evolve for the time $t_{i g n}=3 \tau_{e d}$, and in S3 for the time $t_{i g n}=2 \tau_{e d}$, to develop the steady-state turbulent flow field. At the time $t_{i g n}$ a planar laminar flame with its front parallel to the $x-y$ plane was imposed in the domain. The initial flame position $z_{T, 0}$, given in Table 2 , was assumed to be the location of the $Y=0.5$ point in the exact laminar flame solution for the reaction model parameters and fuel conditions used. Values of $\rho, P$, and $Y$ in each cell were reset to those obtained from the laminar flame structure based on the cell-center $z$-coordinate. Combustion product is located toward the left $z$-boundary and fuel is located toward the right $z$-boundary. The velocity field was not modified, thereby preserving the structure that developed during the equilibration stage. Hereafter, for simplicity we refer to the moment of ignition as $t=0$.

Prior to $t_{i g n}$, all domain boundaries are periodic. At $t_{i g n}$, boundary conditions (BCs) along the left and right $z-$ boundaries are switched to zero-order extrapolation. As the flow evolves, it develops a complex pattern near the boundaries. Local turbulent motions may have velocity vectors directed both in and out of the domain at various points along the boundary. Moreover, this is superimposed on the large-scale net flow associated with the expansion of burnt material. Consequently, BCs must provide the possibility for the fluid to move both in and out of the domain while adjusting to the flow. We find that zero-order extrapolation BCs successfully manage to accomplish this goal while preventing both any unphysical pressure build-up in the domain and the formation of artificial large-scale rarefaction waves at the boundaries.

This type of BCs is known to cause reflections of acoustic perturbations. High-speed turbulent flow in the domain, however, itself generates substantial acoustic noise and even weak transonic shocklets. These features are substantially stronger than reflections from the boundaries, so that it becomes nearly impossible to distinguish such reflected perturbations from those generated by the flow field itself. It would, nevertheless, be desirable in the future to investigate the effects of other types of BCs on the system evolution in order to assess its sensitivity to the prescribed BCs.

Fluid entering the domain with the large-scale inflow does not initially have the proper Kolmogorov-type spectrum. Provided that the turbulent flame brush is located sufficiently far from the boundaries, the typical time for the fluid to travel from the boundary to the flame is longer than a few $\tau_{e d}$. Therefore, this time is sufficient for the incoming flow to be subjected to the external driving in the domain and to develop the equilibrium spectrum.

Finally, given the simulation parameters discussed above and listed in Table 2] it is instructive to consider the location of the regime, studied in this work, in the traditional combustion diagrams. Figure 2 shows the two most widely used examples of such diagrams, namely those described by Peters [1] and Williams [2, 3]. Detailed discussion of the diagrams including various lines delineating different combustion regimes are given in [1, 2, 3].

It is important to emphasize that the laminar flame width, $l_{F}$, used in the Peters' diagram, is different from the thermal flame width, $\delta_{L}$, used throughout this paper as a characteristic length scale associated with the laminar flame. In particular, $l_{F}$ is defined as

$$
l_{F}=\frac{D}{S_{L}}
$$


where $D$ is the characteristic diffusion coefficient, and it is assumed that $L e=\operatorname{Pr}=S c=1$. The value of $D$, however, is not constant and varies with temperature (cf. eq. (6)). Thus, the question arises concerning the value of $T$ that should be used to define $l_{F}$. It is reasonable to take the temperature and density in the exact laminar flame profile that correspond to $Y=0.5$. This value of $Y$ represents the boundary between the preheat and reaction zones. Moreover, in the vicinity of this point, $T$ has the steepest gradient which is used to define $\delta_{L}$. Then, using the corresponding values $T=1212 \mathrm{~K}$ and $\rho=2.1 \times 10^{-4} \mathrm{~g} / \mathrm{cm}^{3}$ in eq. (6), we find that $l_{F} \approx 2 \delta_{L}$, which is approximately equal to the full width of the laminar flame. For consistency, we use this value of $l_{F}$ in both diagrams as a characteristic scale of the laminar flame.

The Damköhler and Reynolds numbers, as well as the Gibson scale are then defined in the usual manner [1]

$$
D a=\frac{l S_{L}}{l_{F} U_{l}}, \quad R e=\frac{l U_{l}}{l_{F} S_{L}}, \quad L_{G}=l\left(\frac{S_{L}}{U_{l}}\right)^{3}
$$

Using these equations, values of $\delta_{L}$ and $S_{L}$ from Table 1, and $l$ and $U_{l}$ from Table 2, the location of the regime considered here is shown in the combustion diagrams (Fig. 2). Simulations, presented here, fall in the region that is transitional between thin and broken reaction zones. In this regime, turbulent transport is expected to become comparable to the molecular diffusive processes and, thereby, to start affecting the internal flame structure not only in the preheat but also in the reaction zone. Such break-up of the laminar structure of the flamelets would mark the onset of the distributed mode of burning (Fig. 2, right panel). Note also that the Gibson scale, $L_{G}$, is almost four orders of magnitude smaller than $\delta_{L}$ (Table 2). This also suggests that turbulent cascade should penetrate deep inside individual flamelets disrupting them. The performed simulations allow us to investigate the validity of such classification of this combustion regime.

\section{Results}

\subsection{Overall evolution and global properties of the flame brush}

Upon ignition, the planar laminar flame starts being wrinkled by turbulent motions, and the turbulent flame brush gradually develops. Within $\sim 2 \tau_{e d}$, the flame brush reaches a quasi-steady state in which its width $\delta_{T}$ and speed $S_{T}$ on average remain constant.

Figure 3 shows the 3D structure of the turbulent flame brush based on the distribution of the fuel mass fraction. A general examination of the flame morphology in Fig. 3 already suggests crucial similarities as well as differences in the system at different resolutions. In all three simulations, the flame brush represents a highly convolved flame with a thinner reaction zone and a thicker preheat zone. The thin black line on the sides of the computational domain marks the boundary between the preheat and reaction zones, and the thin white line shows the location of the peak reaction rate. Typically, these two lines closely follow each other. The preheat zone, shown in green and blue, is much wider than the reaction zone, and generally its shape does not follow that of the reaction zone.

The flame surface, when viewed from the product side, appears remarkably independent of resolution. In all cases, it is smooth on smaller scales and curved on larger scales comparable to the turbulent integral scale. In contrast, the flame surface on the fuel side is wrinkled on progressively smaller scales with increasing resolution. This suggests that turbulent motions on smaller scales affect the flame structure in the preheat zone and their effect becomes less pronounced with increasing temperature toward the reaction zone.

Figure 4 shows the evolution of $\delta_{T}$ and $S_{T}$, normalized by $\delta_{L}$ and $S_{L}$. Hereafter, we define the width of the turbulent flame brush as

$$
\delta_{T}=z_{1, \max }-z_{0, \min },
$$

where $z_{0, \min }$ is such that $Y<0.05$ for all points $(x, y, z)$ which have $z<z_{0, \min }$ and $z_{1, \max }$ is such that $Y>0.95$ for all points $(x, y, z)$ which have $z>z_{1, \max }$. In other words, $z_{0, \min }$ marks the rightmost $x$ - $y$-plane to the left of which is pure product, while $z_{1, \max }$ marks the leftmost $x$-y-plane to the right of which is pure fuel. This is illustrated in Fig. 5.

There are several different ways to define the turbulent burning velocity, as described in detail in [5]. The flame brush propagates into the fuel and its total net displacement velocity is a function of both the global fuel-consumption speed as well as the net fuel velocity in the domain. The net fuel velocity depends sensitively on many factors, such as the details of the flow at the moment of ignition, subsequent evolution of the stochastic turbulent field, and conditions 
at the domain boundary. Consequently, the speed of the flame-brush displacement with respect to the grid can vary, and it cannot be predicted a priori.

Since we are considering an ideally infinite, unbounded system, there are no boundaries or obstacles with respect to which the velocity of the flame brush could be defined. At the same time, displacement velocity with respect to the laboratory reference frame, associated with the computational mesh, does not have physical significance. In principle, we could associate a stationary reference frame with the fuel located (infinitely) far from the flame brush. Such fuel, while being in the state of turbulent motion, could be considered stationary on average, and the displacement velocity of the flame brush with respect to it could be defined. In practice, however, the volume of fuel between the flame brush and the domain boundary is not sufficiently large to allow us to perform averaging that would eliminate spatial and temporal variability in the fuel velocity and would provide a reliable inertial reference frame. [5])

Therefore, we define the turbulent flame-brush velocity $S_{T}$ as the global fuel consumption speed (cf. eq. (15) in

$$
S_{T}=\frac{\dot{m}_{R}}{\rho_{0} L^{2}}
$$

Here $\dot{m}_{R}$ is the total rate of fuel consumption inside the flame brush, i.e., the total mass of reactants which is transformed to product per unit time. On average, the flame brush is planar in the domain with the cross-sectional area of $L^{2}$. Then in steady state in order for the flame brush to support fuel consumption rate $\dot{m}_{R}$, the fuel must be supplied to the flame through the area $L^{2}$ at the rate $\rho_{0} S_{T} L^{2}$. Note that even though the fuel density varies locally by a small amount due to the compressibility in the flow, on average it remains equal to the original value $\rho_{0}$. Therefore, in a perfectly steady state, $S_{T}$ represents both the fuel consumption speed as well the velocity of the flame-brush displacement with respect to stationary fuel far from the flame brush.

Table 3 lists time-averaged values of $\delta_{T}$ and $S_{T}$. Time averaging is performed over 14 large-scale eddy turnover times. The start of the averaging interval is chosen as $t=2 \tau_{e d}$, when the system enters the quasi-steady state. While the particular moment of the onset of this state is not precisely defined, we find that the flame-brush parameters, discussed in this paper, reach their time-averaged values by $2 \tau_{e d}$.

Even the relatively high-speed turbulence, considered here, only increases $S_{T}$ rather modestly, with the value of $S_{T}$ on average saturating at $\approx 4 S_{L} \approx 12 \mathrm{~m} / \mathrm{s}$. The flame-brush width in S3 is somewhat larger than the domain width, namely $\overline{\delta_{T}}=1.8 \mathrm{~L}$, and it is 7.7 times larger than the turbulent integral scale $l$.

Table 3 also lists the order of self-convergence of each parameter. Since the computational cell size decreases progressively by a factor of 2 between each simulation, the order of self-convergence $O(\phi)$ of a parameter $\phi$ is defined as

$$
O(\phi)=\log _{2}\left(\frac{\left|\phi_{S 1}-\phi_{S 3}\right|}{\left|\phi_{S 2}-\phi_{S 3}\right|}\right) .
$$

The table shows that both $\delta_{T}$ and $S_{T}$ converge quadratically, as would be expected for a second-order numerical method.

In a dynamic unsteady flow, fuel consumption inside the flame brush is not perfectly balanced by the influx of fresh fuel. Inflow of fuel can dominate as the flame brush increases its width and the amount of fuel inside. This is followed by a period when fuel consumption prevails, rapidly burning the accumulated reactants and causing the flame brush to shrink in size. While on average those two processes do remain balanced maintaining constant average width and speed of the flame brush, such variability is an important part of the overall flame-brush evolution. Figure 4 shows that in the lowest resolution case $\mathrm{S} 1, \delta_{T}$ varies by more than a factor of two, and $S_{T}$ varies by more than a factor of three. Furthermore, the overall variability decreases with increasing resolution. This is particularly apparent for the turbulent flame speed. Note that peak values of $S_{T}$ in S3 are $\lesssim 6$, while in S1 they reach $\approx 14$ in one episode.

Figure 3, which shows the flame-brush structure in all three simulations at $t=13 \tau_{e d}$, also illustrates such variable nature of the system. Figure 4 shows that at this time, S1 and S3 undergo two extreme episodes in their evolution. The flame brush in S1 reaches its maximum width, which is $\sim 50 \%$ larger than its average value, while $S_{T}$ increases by more than a factor of two. S3, on the other hand, is in the most quiescent phase with $\delta_{T}$ shrinking by one third and $S_{T}$ being only twice the laminar flame speed. Finally, S2 at this time is in its average state with both $\delta_{T}$ and $S_{T}$ very close to their average values. Therefore, aside from differences in the preheat-zone structure, the three states of the turbulent flame shown in Fig. 3 represent the main stages in the flame-brush evolution which are characteristic of all three simulations. Namely, they illustrate recurring transitions between periods of a widened flame brush, containing 
highly convolved flame surface, and more quiescent stages, when the thinner flame brush contains a smoother, flatter flame.

Qualitatively, the nature of such variability can be explained as follows. The internal structure of the flame brush is determined by two counteracting processes. On one hand, flame surface area is created by turbulent motions bending and stretching the flame and, thereby, increasing both the flame brush thickness and the flame surface density inside the brush. On the other hand, this process is balanced by flame-surface destruction, which is an inherently nonlinear stabilization process preventing unbounded growth of the flame perturbations [32, 33]. Flame-surface destruction takes place in regions where the curvature radius of the flame surface becomes comparable to the flame thickness ("cusps") or where the flame sheets come close to each other. Such a picture of the balance between surface creation and destruction has been reflected in a number of analytical and numerical models of turbulent flames (e.g., see [34, 35] as well as the review by [5] and references therein).

Three distinct stages of the destruction process can be seen in the upper and middle panels of Fig. 3 , Consider region $\mathrm{A}$, where the flame surface is tightly packed by turbulence. This results in two flame sheets being so close to each other that their preheat zones start to overlap. Moreover, each flamelet is also curved on scales comparable to its thickness. Thermal flux from hot products into the colder fuel becomes focused in such cusps, thus accelerating the heating of reactants and effectively increasing the local burning velocity. This makes the two flame sheets propagate faster toward each other.

Eventually, this may lead to the configuration seen in region B. There preheat zones of each flame sheet substantially overlap even though the reaction zones are still separated. At this point, the whole region acts as one large cusp with the heat flux coming from all directions and thus rapidly heating up the unreacted material. The temperature quickly reaches the ignition point and all of this region becomes a reaction zone.

Examples of such merged, extended reaction zones can be seen in regions $\mathrm{C}$. Those parts of the flame quickly burn out, which reduces the curvature and the overall surface area of the flame. Such events are transient phenomena, and they must not be mistaken for stable distributed reaction zones. In particular, it is very important in experimental settings for the diagnostics to be able to properly characterize such broad reaction zones as transients.

In fast turbulence, as present in these simulations with $D a \ll 1$, the characteristic turbulent time $\delta_{L} / U_{\delta}$ at the scale of the laminar flame width is much smaller than the laminar flame self-crossing time $\delta_{L} / S_{L}$. Therefore, turbulence can bring two flame sheets together and then pull them apart before each one propagates over the distance $\sim \delta_{L}$. As a result, flame sheets in region A in Fig. 3 can be separated by turbulence before they ever merge. On the other hand, the local burning velocity in cusps can be very large, in fact, it could in principle be infinite. Therefore, once a cusp is formed, i.e., there exists a region of significant flux focusing, it burns out on time scales short enough for turbulence to have little or no effect on it.

In summary, periodically the flame brush undergoes a state in its evolution when turbulence rapidly increases the flame surface which becomes highly convolved and develops cusps. This situation is represented by the upper panels in Fig. 3. The local burning velocity in cusps increases substantially to the point when turbulent motions are no longer locally dominant. This causes cusps to burn out quickly, leading to an increased rate of global fuel consumption and smoothing the overall flame surface. This stage is shown in the middle panels in Fig. 3 Eventually, this results in a flame brush which is substantially thinner and which contains much less convolved flame surface, as seen in the bottom panels of Fig. 3 Such flatter, slower flame with fewer cusps now again becomes susceptible to the action of turbulent motions, which can increase its surface area, and, hence, the cycle repeats.

Finally, Fig. 6 shows temperature structure in the flame brush in S2, corresponding to the middle panels of Fig. 3 , As expected, the temperature distribution closely follows that of the fuel mass fraction.

\subsection{Internal structure of the flame brush}

Figure 3 suggests that even in the presence of high-speed turbulence, the flame brush consists of highly convolved flamelets. In order to examine this, we need to identify such flamelets and determine their internal structure, if they are indeed present. To accomplish this, we developed the following method.

The range of values of $Y$ and $T$ in the domain is discretized into $n$ equal intervals. Typically, we choose $Y \in$ $[0.01,0.99]$ and $T \in[350.0,2135.0] \mathrm{K}$. The upper bound of the temperature interval is the adiabatic flame temperature $T_{P}$. The lower bound is somewhat higher than $T_{0}$. In the course of the simulation, fuel temperature away from the flame slowly rises due to the turbulent energy dissipation, thereby raising the minimum temperature value in the 
domain (see $\$ 2.4$ ). The lower bound of the temperature interval is chosen to account for the heating of the fuel and to include only the temperature increase associated with flame propagation. For each of the discrete values of $Y_{i}$ and $T_{i}$, with $i$ taking values 0 to $n$, we construct an isosurface $\mathcal{S}_{i}$ with area $A_{i}$ using the "marching cubes" algorithm. We also determine the volume $V_{i}$ bounded by the isosurfaces $\mathcal{S}_{i}$ and $\mathcal{S}_{i+1}$. Since the flame brush always intersects the computational domain boundaries, this volume will always be bounded by $\mathcal{S}_{i}, \mathcal{S}_{i+1}$, and the boundaries. Then we construct the expression

$$
\eta_{i+1} \equiv V_{i} / A_{i}
$$

assuming $\eta_{0}=0$. The $\eta_{i+1}$ gives the average distance separating all points in the domain in which $Y=Y_{i+1}$ and $T=T_{i+1}$ from those in which $Y=Y_{i}$ and $T=T_{i}$ respectively. Finally, $Y(\eta)$ and $T(\eta)$ give the average flamelet structure in the flame brush. In Appendix Appendix A we discuss in detail the properties and limitations of this method, as well as the issues associated with its practical implementation.

For finite values of $\Delta Y \equiv Y_{i+1}-Y_{i}$ or $\Delta T \equiv T_{i+1}-T_{i}$, the local separation between the two isosurfaces $\mathcal{S}_{i}$ and $\mathcal{S}_{i+1}$ will vary from point to point. Consider Fig. 3. Thin white and black lines mark isosurfaces $Y=0.2$ and $Y=0.6$. Regions can be identified where they come very close to each other and where they are substantially separated, e.g., as in regions $\mathrm{C}$ (see also Fig. 5]. The same is also true for temperature, as evidenced by Fig. 6 We show in Appendix Appendix A however, that as $\Delta Y$ and $\Delta T$ become smaller, the variation in the local separation of the two isosurfaces will also decrease.

Therefore, $\eta_{i+1}$, as defined in eq. (15), can be considered as a distance between consecutive isosurfaces $\mathcal{S}_{i}$ and $\mathcal{S}_{i+1}$ averaged over their entire area. Regions of large flame stretch, in which the flamelet becomes very thin, would tend to decrease the value of $\eta$. On the other hand, cusps, regions of significant flame folding, and broadened reaction zones would tend to make $\eta$ larger. Thus, the flame structure $Y(\eta)$ and $T(\eta)$, produced by this analysis for a given instantaneous state of the flame brush, is an average of all local realizations of the flame structure at each point of the flame surface.

Subsequently, $Y(\eta)$ and $T(\eta)$ can be time-averaged, as a substitute for ensemble-averaging, to obtain the statistically dominant structure of the flamelets inside the flame brush. The resulting structure then contains information about the statistical weight of cusps and broadened reaction zones in the ensemble of all possible flamelet configurations that can exist for given conditions. Similarity of this structure to that of the planar laminar flame would indicate that cusps and broadened reaction zones are indeed transient phenomena, and they do not dominate the internal flamebrush structure. On the other hand, if substantial flame broadening by turbulent transport indeed takes place, as would be expected in the broken reaction-zones regime, then broadened reaction zones would dominate the statistical ensemble of possible flame configurations, and the resulting averaged structure would be significantly wider than the laminar flame profile.

We applied this method to the flow in simulations S1 $-\mathrm{S} 3$. For S1 and S2, $n=\delta_{L} / \Delta x$, as given in Table 2 , in accordance with the criterion in eq. A.6. For S3, however, $n$ was limited to a lower value, namely 16 , due to the high computational cost of reconstructing a substantially larger number of isosurfaces 1 in the case of $n=32$. The $Y$ and $T$ profiles were averaged over the time interval $(2-16) \tau_{e d}$. In particular, averaging was performed over 401 discrete time states in S1, 101 in S2, and 419 in S3. The resulting profiles are shown in Fig. 7

Values of the $x$-coordinate in the figure are chosen such that $Y(x=0)=0.5$ both in the exact laminar flame solution and in the obtained profiles. In the exact solution, this also uniquely determines the position of the temperature profile since there is a precise relation between $T$ and $Y$. At the same time, the method we are using to reconstruct the flamelet structure does not provide any means to relate the obtained distributions of different variables, as it is discussed in detail in Appendix Appendix A. Values of $\eta$ are independent in each profile. It is not a common spatial coordinate, and it simply indicates distance between consecutive isosurface values of $Y, T$, etc. Each profile is a statistically averaged representation of all existing flamelet configurations in the flame brush. Thus, any relation between the $Y$ and $T$ profiles can only exist in the statistical sense, namely such relation would only indicate the most likely values of $T$ for given values of $Y$ and vice versa. Therefore, here for simplicity we offset temperature profiles so that $T(x=0)$ would correspond to $\left(T-T_{0}\right) /\left(T_{P}-T_{0}\right)=0.5$, as in the exact laminar flame solution.

\footnotetext{
${ }^{1}$ Due to the adaptive nature of the algorithm, which implements the reconstruction method as described in Appendix Appendix A the actual number of isosurfaces and isovolumes that need to be reconstructed for each variable can be significantly larger than $n$. In particular, for $n=32$ on average more than 100 isosurfaces need to be reconstructed for each time state with their total number exceeding 50,000.
} 
The principal conclusion that emerges from Fig. 7 is that, on average, the turbulent flame brush interacting with high-speed turbulence represents highly convolved flamelets that have internal structure of a laminar flame with a somewhat broadened preheat zone. The $Y$ and $T$ profiles are very close to the exact laminar solution in the reaction zone. They begin to diverge from the laminar profiles only at the outer boundary of the reaction zone, around $x / \delta_{L} \approx$ $0.1-0.2$. In fact, the $Y$ profile for S3 is virtually indistinguishable from the laminar solution inside the reaction zone. The remarkable similarity of the flamelet structure inside the reaction zone to that of the planar laminar flame is a partial justification for our choice of the $x$-coordinate origin for the temperature profile.

The deviation of the $Y$ and $T$ profiles from the laminar solution increases with distance from the reaction zone. Even for such strong turbulence, however, this difference is fairly small, with the total preheat zone width being increased by less than a factor of two.

The profiles, obtained for all three simulations, are very close to each other for all values of both $Y$ and $T$. Given the statistical nature of the distributions, this serves as evidence that the simulations can be considered converged. More importantly, the agreement between profiles in the preheat zone shows that thermal conduction is enhanced predominantly by turbulent motions on scales $\sim \delta_{L}$. The amount of energy contained on this scale is the same in all simulations (see Fig. 1). On the other hand, the energy contained on smaller scales, and, thus, the velocity, increases substantially from case S1 to S3. If those scales were to contribute noticeably to the overall thermal transport, such increase in energy content on those scales would be reflected in a broader preheat zone. There is, however, no evidence of this.

Finally, an important question concerns the degree of variability of instantaneous distributions of $Y$ and $T$ in the course of system evolution. In particular, this question is crucial for determining the statistical significance of the observed preheat zone broadening in the time-averaged flame structure.

It was discussed above that $\eta$ in the distributions $Y(\eta)$ and $T(\eta)$ is simply the distance between two consecutive isosurface values rather than a spatial coordinate with respect to some fixed reference frame. Therefore, in order to compare instantaneous profiles of $Y(\eta)$ or $T(\eta)$, all values of $\eta$ must be referred to some common point in which all profiles will, thereby, coincide. The choice of such reference point is arbitrary and while it does not affect the shape of the resulting time-averaged distribution, it changes the range of variations of individual profiles. Overall, such variations must be considered only in the vicinity of the chosen reference point. Any difference in the shape of the profiles near this point will cause the separation between profiles to increase with distance from it, thereby, magnifying the perceived variability in the profile shape.

In the case of S3, such variability range is shown in Figs. 7. 8 with profiles for all individual time states contained within the shaded gray areas. All profiles were chosen to coincide at the boundary between the reaction and preheat zones (Fig. 7$\}=0.5$ and $\left(T-T_{0}\right) /\left(T_{P}-T_{0}\right)=0.5$ ), at the point of peak reaction rate (Fig. 8 a, $Y=0.15$ and $\left.\left(T-T_{0}\right) /\left(T_{P}-T_{0}\right)=0.85\right)$, and in the preheat zone (Fig. $8 \mathrm{~b}, Y=0.85$ and $\left.\left(T-T_{0}\right) /\left(T_{P}-T_{0}\right)=0.15\right)$.

Overall, there is extremely small variability in the flame structure both in the reaction zone (Fig. 8 a) and in the steepest region of the profiles (Fig. 7) throughout the time interval used for averaging. This supports our choice of this interval as a period of quasi-steady state in the system evolution. There is, however, substantially more variability in the preheat zone (Fig. $8 \mathrm{~b}$ ). Moreover, variability of the fuel mass fraction there appears to be somewhat larger than that of the temperature.

Figure $8 \mathrm{~b}$ shows that in the preheat zone the laminar solutions marginally lie outside the variability range of the profiles. This confirms that the observed broadening is statistically significant, and such flamelet structure is prevalent throughout the evolution of the system. On the other hand, the fact that the laminar solution is so close to the boundary of the variability range shows how weak this effect is, even in the case of such strong turbulence as is present in these simulations.

\section{Discussion and Conclusions}

We have explored the dynamics and properties of a turbulent flame formed in the presence of driven subsonic, highspeed, homogeneous, isotropic Kolmogorov-type turbulence in an unconfined system, i.e., in the absence of walls and boundaries. The Damköhler number for the turbulent flow is $D a=0.05$ while the Gibson scale is $L_{G}=2.96 \times 10^{-4} \delta_{L}$.

Numerical modeling was performed using the massively parallel, fully compressible, higher-order, dimensionally unsplit, reactive-flow code Athena-RFX [19]. The highest-resolution calculation S3 required approximately 100,000 
CPU hours on 1024 processor cores of the Ranger platform at TACC, and a similar amount of computational time was necessary for data processing and reconstruction of the flamelet structure.

\subsection{General properties of the turbulent flame}

Our results show that the turbulence-flame interaction leads to the formation of a steadily propagating turbulent flame brush. We followed the system evolution in this quasi-steady state for 14 large-scale eddy turnover times, which was sufficient to provide long-term statistics. On average, both the flame width and speed remain constant, even though at each moment in time they can vary substantially. We observed an increase in the time-averaged turbulent flame speed, $\overline{S_{T}}$, compared to the laminar flame speed, $S_{L}$, by a factor of 4 . The time-averaged turbulent flame width, $\overline{\delta_{T}}$, was almost twice the domain width and the energy injection scale, $L$. It was also almost 8 times larger than the turbulent integral scale, $l$.

An important question concerns the parameters which predominantly determine $\delta_{T}$. In our simulations, the system size, which is set by the domain width, is equal to $L$, and, thus, it is close to $l$. It is, therefore, impossible to separate the individual role played by the system size, $L$, and $l$ in defining the equilibrium value of $\delta_{T}$. In particular, given fixed values of $L$ and $l$, it is not clear whether a substantial increase in system size would result in a change in $\delta_{T}$. Moreover, it is not clear whether in such a large system there would still exist the observed steady flame propagation with a fixed width that is much smaller than the system size. Finally, an important question concerns the relation between $L$ and $\delta_{T}$. Does an increase in $L$ lead to a corresponding increase in $\delta_{T}$, or is there a limiting value of $\delta_{T}$ ?

The study by Aspden et al. [15], which used faster turbulence relative to $S_{L}$ than this work, also observed a turbulent flame propagating with a steady width and speed. In that work energy as well was injected at the scale close to the domain width. In their calculation that had the highest ratio of the turbulent velocity to $S_{L}$, the domain size relative to the laminar flame width, $\delta_{L}$, however, was almost 8 times larger than in our case, namely $L \approx 65 \delta_{L}$ vs. $L=8 \delta_{L}$. Yet that simulation produced $\delta_{T} \approx 3 L$, which is close to that obtained in our simulations, namely $\delta_{T} \approx 2 L$. This suggests that the width of the flame brush increases with the increase in the driving scale and system size. Their work, however, as well does not address the question of the individual role of these two scales.

In systems which are much larger than the driving scale, most likely both $L$ and $l$, rather than the domain size, play the primary role in setting the equilibrium width of the flame brush. Indeed, the turbulent integral scale along with the driving scale are the characteristic scales of coherent turbulent motions. Moreover, on scales $\lambda \gg L$, the turbulent energy spectrum has the $k^{4}$ dependence [36]. Therefore, the energy contained in those large scales decreases rapidly with increasing scale, which minimizes their potential role in defining the turbulent flame width. At the same time, in situations when the domain width is close to $L$, domain boundaries imprint an artificial periodicity on the flow. This can constrain the growth of $\delta_{T}$ and, thus, set its equilibrium value.

The main reason for the difficulty with addressing these issues is the limited range of spatial scales presently accessible even in the most heroic numerical calculations. The need to provide sufficiently high resolution at the scale of the laminar flame, and still maintain some separation between $L$ and $\delta_{L}$, does not allow the system size to be more than an order of magnitude larger than $L$. This is insufficient to provide a conclusive answer to the questions mentioned above.

\subsection{Internal structure of the flame brush and the role of small-scale turbulence}

A major conclusion of the simulations presented in this paper is that in the presence of high-speed turbulence the flame brush is comprised of highly convolved flamelets. The time-averaged internal structure of those flamelets is very close to that of the planar laminar flame, and, in fact, both structures are virtually identical in the reaction zone. The preheat zone, however, does show evidence of broadening, although this effect, while statistically significant, is fairly small with the width of the preheat zone increasing by less than a factor of two.

The internal structure of flamelets inside the flame brush was reconstructed directly by a method based on the isosurfaces of the 3D distribution of a scalar quantity, such as fuel mass fraction, $Y$, or temperature, $T$. The overall method does not make a priori any assumptions concerning the possible underlying flame structure or even the presence of the flame itself.

The absence of any broadening in the reaction zone indicates that combustion takes place in the thin reaction zone regime. Turbulent velocities on the scale of the laminar flame width are 15 times larger than the laminar flame speed. In principle, this would suggest that the turbulent transport should overwhelm the molecular thermal conduction 
and species diffusion. It appears, however, that disrupting the flame would require substantially higher turbulent intensities. This result is in general agreement with the behavior observed in [10, 15].

According to the traditional combustion-regime diagrams, shown in Fig. 2, some broadening of the reaction zone would formally be expected in the regime considered here. For instance, according to the Williams' diagram (Fig. 2 , right panel) the simulations are in the broken reaction-zone regime, while according to the Peters' diagram (Fig. 2 , left panel), they fall almost exactly at the boundary of thin and broken reaction zones. The $K a_{\delta}=1$ line in the Peters' diagram is typically constructed based on the assumption that the reaction-zone width is $1 / 10$ of the full flame width. In our case such estimate is too conservative with this fraction being $\sim 1 / 3-1 / 2$ (cf. Fig. 7). This would lower the $K a_{\delta}=1$ line, thereby, also placing simulations S1 - S3 in the broken reaction-zone regime. Of course, given the approximate nature of such diagrams, boundaries between various combustion regimes are not really precisely defined and, instead, they should rather be viewed as the transition areas. Nonetheless, the fact that our results do not show any evidence of the reaction-zone broadening suggests that the range of the regimes in which flamelets exist may be substantially larger, even when the uncertainties in the precise location of the indicated regime boundaries are taken into account.

The obtained flamelet structure represents the statistically dominant state of flamelets inside the flame brush. At the same time, substantially broadened reaction zones can arise in the flow as a result of collisions and mergers of flame sheets or in the regions of high flame curvature (cusps). Such broadened reaction zones, however, are transient in nature being the result of the rapidly changing $3 \mathrm{D}$ configuration of the flame surface. They should not be considered as evidence of a distributed mode of burning since they are in no way supported by turbulent transport.

The progressive increase in resolution in calculations S1 - S3 results in a substantial increase in the energy of turbulent motions on scales $\lambda \lesssim \delta_{L}$ and causes the energy cascade in nonreactive turbulence to extend to much smaller scales. This, however, is insufficient to disrupt the internal structure of the reaction zone, which is demonstrated by the fact that the $Y$ and $T$ profiles, describing the internal structure of the flamelets in all three simulations, are very similar for all values of $Y$ and $T$. Furthermore, the structure of the broadened preheat zone does not change between $\mathrm{S} 1$ and S3 suggesting that such broadening is determined by scales $\lambda \gtrsim \delta_{L}$ rather than small-scale motions. The effect of small-scale turbulence appears only in the progressively finer wrinkling of the flame surface on the fuel side in the coldest part of the preheat zone.

These results lead to the following important conclusion. The turbulent energy cascade fails to penetrate the internal structure of the flame on scales much smaller than $\delta_{L}$, even in the presence of high-speed turbulence considered here. This shows that the response of the flame to the action of turbulence is qualitatively different from that of a passively advected scalar, which can generally be a source of only limited insight into the behavior of the turbulent flames.

This also suggests that the traditional definition of the Gibson scale, $L_{G}$, cannot serve as a useful indicator of the efficiency with which small-scale turbulence penetrates the flame. In particular, in our simulations $L_{G}$ is almost four orders of magnitude smaller than $\delta_{L}$ (see Table 2) which, however, is not reflected in any appreciable effects of small-scale turbulence on the flame.

What mechanisms are responsible for suppressing the effects of small-scale motions in the reactive turbulence? The dramatic difference in the degree of wrinkling of the flame surface on the fuel and product sides, observed in the highest resolution case S3, suggests that this suppression of small-scale turbulence occurs as the flow passes through the individual flamelets rather than the whole flame brush. Two possible processes, which agree with this observation, were previously suggested (see discussion in [5] and references therein). On one hand, as fluid passes through the flame, it heats up and expands. Since the circulation of individual eddies must be conserved during this expansion, the rotational velocity of eddies must decrease. Moreover, this will also shift energy from smaller to larger scales. On the other hand, such expansion causes rapid acceleration of the fluid in the reference frame of the flame brush. As a result, the residence time of individual eddies inside the flame brush decreases as the fluid quickly leaves the flame brush. It is not clear, however, whether these two mechanisms are sufficient to completely account for the observed behavior. It is also not known whether their effect on small and large scales is similar or not. Answering these questions is important for our understanding of the turbulence-flame interaction.

Ultimately, our understanding of the turbulent combustion process is measured, to a large extent, by our ability to predict the turbulent burning velocity. According to the Damköhler concept [4] discussed in $\S[1$ two processes can determine the turbulent flame speed: increase in the flame surface area by turbulent stretching and folding of the flame, and increase in the local flame speed by turbulent diffusion. 
Similarity of the internal flame structure to that of the planar laminar flame shows that the local flame speed cannot be substantially increased by turbulence. The flame structure is determined by the balance of the reactions and the diffusive processes. In our model, the reaction rate is determined purely by the local thermodynamic state of the fluid and, thereby, it is not directly affected by turbulence which can only enhance the diffusive transport. For a given one-step Arrhenius reaction model, the coefficients of thermal conduction and species diffusion uniquely determine both the flame structure and speed. If turbulence does indeed increase the effective diffusive coefficients, this would increase the local flame speed, but this would also change the flame structure. We do not find any evidence of this.

This raises the question whether the observed turbulent flame speed, shown in Fig. 4 $4 \mathrm{~b}$, can be fully accounted for only by the increase in the flame surface area or whether other processes contribute to it. We will discuss this issue in detail in a separate paper [37].

Finally, it is important to emphasize that averaged distributions of various quantities, such as $Y, T$, or the reaction rate, $R$, through the turbulent flame do not provide information about the internal structure of the flame brush, and they cannot be viewed as evidence of flame broadening. Consider Fig. 9, which shows the $x$-y-averaged profiles of $Y$, $T$, and $R$ along the $z$-axis in simulation $\mathrm{S} 3$. All profiles, including that of the reaction rate, are smooth and the overall structure is similar to the one shown in Fig. 7, with the key exception that the width of the profiles is substantially larger by a factor $\approx 4$. The broad, smooth profile of the reaction rate can lead to the erroneous conclusion that this system represents a distributed mode of burning with a substantially broadened reaction zone. If the flame brush volume is large enough, then the average profiles of $Y, T$, etc., will always be smooth and wide, since they are simply the result of averaging many individual flamelet structures. Moreover, the turbulent flame is the region in which $Y$ and $T$ transition from their values in the fuel to those in the product. Therefore, those profiles smoothly and monotonically change from their fuel to product values, and the averaged distribution of $R$ simply varies accordingly.

\subsection{Convergence and resolution}

What do our results say about the minimum resolution needed to provide a converged solution in simulations of high-speed turbulent combustion? Based on Table 3 time-averaged values of $\delta_{T} / \delta_{L}$ and $S_{T} / S_{L}$ converge quadratically. The values of $\overline{\delta_{T}} / \delta_{L}$ vary only by $\approx 3 \%$ in the two highest-resolution simulations, $\mathrm{S} 2$ and $\mathrm{S} 3$, while $\overline{S_{T}} / S_{L}$ varies by $10 \%$. Profiles of $Y$ and $T$, which represent the internal flamelet structure, also demonstrate excellent convergence.

The only exception to this converged behavior is the degree of flame-surface wrinkling on the fuel side. It was discussed in $\$ 2.4$ that simulations with different computational cell sizes do not represent the same physical system. The spectral energy distribution in the domain changes with increasing resolution because the amount of energy contained on smaller scales grows. This causes isosurfaces in the coldest regions of the preheat zone to be wrinkled on progressively smaller scales (see Fig. 3). In this context, convergence of the full system to a unique solution in the purely numerical sense would not be expected in such turbulent flows. At the same time, the observed convergence of all key characteristics of the turbulent flame simply means that such small scale motions in cold fuel do not affect the evolution of the flame brush.

Based on these observations, we conclude that a minimum resolution of 16 cells per $\delta_{L}$ is required to capture the evolution of the turbulent flame adequately. Lower resolutions tend to exaggerate both the values and the degree of variability of all major quantities, primarily the turbulent flame width and speed.

A minimum resolution of 16 cells is substantially higher than the resolution of 4 cells per $\delta_{L}$ that is required to reproduce with high accuracy the behavior of the planar laminar flame [19]. This suggests that the need for higher resolution arises due to the turbulent nature of the flow. Our results have shown that small-scale turbulence fails to penetrate the flame and, thereby, to modify both its internal structure and local speed. Indeed, even the resolution of 8 cells per $\delta_{L}$ provided the converged flamelet structure. Therefore, it is rather the multidimensional effects of largescale turbulence and, in particular, the resulting highly curved geometry of individual flame sheets, which require higher resolution.

In the presence of high-speed turbulence, the flame is often folded with a curvature radius comparable to the laminar flame width (see Fig. 3). In such cusps, the Cartesian mesh invariably introduces some degree of unphysical anisotropy to the thermal flux. This effect is not present in the case of a planar laminar flame. In the turbulent flow, however, it causes errors which at low resolutions can become significant in comparison with the overall solution errors. Consequently, higher resolution is necessary to minimize this effect. In order to relax this minimum-resolution threshold and bring it closer in line with that required for a planar laminar flame, better multidimensional coupling of 
diffusive fluxes and their improved coupling with the hydrodynamic fluxes are needed. This is the subject for further work.

\subsection{Implications for an actual stoichiometric $\mathrm{H}_{2}$-air mixture}

The physical model used in this work is based on the first-order Arrhenius kinetics and a simplified reactiondiffusion model of [17] for stoichiometric $\mathrm{H}_{2}$-air mixture. The low computational cost of such approach makes the large-scale multidimensional simulations, such as the ones presented here, computationally feasible. At the same time it also allows us to reproduce accurately the key characteristics of laminar flames in stoichiometric $\mathrm{H}_{2}$-air, such as the laminar flame width and speed, as well as the dependence of these quantities on pressure and temperature.

In order to investigate the role of small-scale turbulence on the flame, we did not include physical viscosity in our model and instead relied on numerical viscosity to provide kinetic-energy dissipation. As a result of this, however, our simulations, strictly speaking, do not describe the behavior of an actual stoichiometric $\mathrm{H}_{2}$-air mixture. In light of this, what can our results say about high-speed turbulent combustion of the actual $\mathrm{H}_{2}$-air?

We have shown that scales $\lambda \ll \delta_{L}$ do not play a pronounced role in determining the dynamics and properties of the turbulent flame brush. This suggests that turbulent motions on yet even smaller scales, which would be present in the cold $\mathrm{H}_{2}$-air mixture, should not be able to change the results either qualitatively or quantitatively. At the same time, the evolution of the turbulent flame is completely determined by scales $\lambda \gtrsim \delta_{L}$. In all our simulations, the inertial range extends to scales smaller than the actual Taylor scale in the product, and it extends to scales smaller than the actual Taylor scale in the fuel in the highest resolution case S3. Therefore, motions on all large scales are properly captured. This fact, along with the negligible role of small scales, suggests that our models can be viewed as representative of the actual behavior of stoichiometric $\mathrm{H}_{2}$-air.

The only exception concerns the wrinkling scale of the flame surface on the fuel side. The presence of turbulent motions on much smaller scales would result in substantially finer wrinkling than shown in Fig. 3] even in our highestresolution simulation. At the same time, the flame surface on the product side is representative of that in the actual reactive mixture.

On the combustion diagrams in Fig. 2 we show the $M a_{F}=1$ line, which separates the regions of subsonic and supersonic turbulence in the cold $\mathrm{H}_{2}$-air fuel under atmospheric conditions. Even under the traditional classification, the potential range of the regimes in which broken or distributed reaction zones would be expected is fairly small. In presented simulations, the turbulent Mach number in cold fuel is $M a_{F}=0.25$. Therefore, in order for the turbulenceflame interaction to remain in the subsonic regime, the turbulent velocity can be increased only moderately. This does not appear to be sufficient, as our results, as well as the results of other studies [10, 15], show that substantially higher turbulent intensities would be required to disrupt the internal flame structure.

While faster turbulence would be more likely to affect the internal flame structure, it would also cause compressibility effects and turbulent heating of the fuel to become much more pronounced. Resulting higher pressures and temperatures would increase the laminar flame speed while decreasing its width, thereby reducing the broadening effect of turbulence. It is also not clear what the effects of the stronger compressive component of the velocity field in such fast turbulence would be. In particular, local compressions and rarefactions can also modify or disrupt the local flame structure. Such processes would be completely distinct from the traditionally considered action of the vortical motions associated with the purely solenoidal part of the turbulent field.

Based on these considerations, the results presented here suggest that the traditional classifications of combustion regimes of stoichiometric $\mathrm{H}_{2}$-air on the basis of the internal flamelet structure and, in particular, the degree of the reaction-zone broadening, do not accurately reflect the actual process of turbulence-flame interaction. In particular, in all subsonic regimes, substantial flame broadening by turbulence appears unlikely, which suggests that the only two possible modes of turbulent stoichiometric hydrogen combustion are the corrugated flamelets and thin reaction zones.

The simplified reaction-diffusion model used in this work cannot capture the full complexity of hydrogen combustion. For instance, the Lewis number of the actual stoichiometric $\mathrm{H}_{2}$-air is less than unity which leads to cellular flames. It will be important for future work to investigate potential effects of the more detailed description of chemical and diffusive processes. This is the subject for future work.

In realistic experimental settings, various aspects of the high-speed turbulence-flame interaction, discussed above, can be further confounded by the effects of the system geometry and the potentially complex nature of the large-scale fluid flow. It is known that turbulent-flame evolution is not "geometry-independent" [5]. The highly anisotropic and 
inhomogeneous turbulence which arises in the presence of strong shear, counter, or jet flows can modify the structure and properties of the turbulent flame. The investigation of the implications of such more complex flows is the subject for a separate study.

We conclude with the question about the universality of the observed behavior. Is it possible to identify a set of parameters which, for the given reactive mixture and flow conditions, would uniquely specify the degree of flame broadening and the role of small-scale turbulence? Are the given ratios of the turbulent intensity and the system size to the laminar flame speed and width, which were used in our simulations, sufficient to apply the obtained results to other fuels?

Our results appear to be in general agreement both with experimental and numerical studies [10, 15] of high-speed turbulent combustion in such different systems as the flat thermonuclear flame in degenerate matter and the jet-burner flame in compressed natural gas. In particular, it appears that, for a broad class of reactive mixtures, the effects of turbulence on the internal flame structure are significantly suppressed, and disrupting the flame requires substantially higher turbulent intensities than can be predicted based on a traditional analysis.

Acknowledgments We are deeply grateful to Vadim Gamezo for numerous valuable inputs in the course of this work. We also thank Craig Wheeler, Forman Williams, James Driscoll, Matthias Ihme, and Ken Bray for stimulating discussions. AYP is particularly grateful to Tom Gardiner for his invaluable assistance with Athena and for his continuing support. AYP also acknowledges the tireless work and invaluable help of the VisIt developers team and especially Hank Childs. This work was supported by the National Research Council Research Associateship Programs in cooperation with the Naval Research Laboratory, by the Office of Naval Research, by the Air Force Office of Scientific Research under the grant F1ATA09114G005, and by the National Science Foundation through TeraGrid resources provided by NCSA and TACC under the grant TG-AST080006N. Additional computing facilities were provided by the Department of Defense High Performance Computing Modernization Program. 
Table 1. Input Model Parameters and Computed Laminar Flame Properties

\begin{tabular}{ccc}
\hline$T_{0}$ & $293 \mathrm{~K}$ & Initial temperature \\
$P_{0}$ & $1.01 \times 10^{6} \mathrm{erg} / \mathrm{cm}^{3}$ & Initial pressure \\
$\rho_{0}$ & $8.73 \times 10^{-4} \mathrm{~g} / \mathrm{cm}^{3}$ & Initial density \\
$\gamma$ & 1.17 & Adiabatic index \\
$M$ & $21 \mathrm{~g} / \mathrm{mol}$ & Molecular weight \\
& & \\
$A$ & $6.85 \times 10^{12} \mathrm{~cm}^{3} / \mathrm{g} \cdot \mathrm{s}$ & Pre-exponential factor \\
$Q$ & $46.37 \mathrm{RT}_{0}$ & Activation energy \\
$q$ & $43.28 \mathrm{RT}_{0} / \mathrm{M}$ & Chemical energy release \\
$\kappa_{0}$ & $2.9 \times 10^{-5} \mathrm{~g} / \mathrm{s} \cdot \mathrm{cm} \cdot \mathrm{K}^{\mathrm{n}}$ & Thermal conduction coefficient \\
$D_{0}$ & $2.9 \times 10^{-5} \mathrm{~g} / \mathrm{s} \cdot \mathrm{cm} \cdot \mathrm{K}^{\mathrm{n}}$ & Molecular diffusion coefficient \\
$n$ & 0.7 & Temperature exponent \\
& & \\
$T_{P}$ & $2135 \mathrm{~K}$ & Post-flame temperature \\
$\rho_{P}$ & $1.2 \times 10^{-4} \mathrm{~g} / \mathrm{cm}^{3}$ & Post-flame density \\
$\delta_{L}$ & $0.032 \mathrm{~cm}$ & Laminar flame thermal width \\
$S_{L}$ & $302 \mathrm{~cm} / \mathrm{s}$ & Laminar flame speed \\
\hline
\end{tabular}

Table 2. Parameters of Simulations ${ }^{\mathrm{a}}$

\begin{tabular}{|c|c|c|c|c|}
\hline & S1 & S2 & S3 & Description \\
\hline $\mathcal{D}$ & $64 \times 64 \times 1024$ & $128 \times 128 \times 2048$ & $256 \times 256 \times 4096$ & Domain grid size \\
\hline $\mathcal{D}_{A}$ & & $1 \times 1 \times 16$ & & Domain aspect ratio \\
\hline$L$ & & $0.259 \mathrm{~cm}=8 \delta_{L}$ & & Domain width, energy-injection scale \\
\hline$\Delta x$ & $4.05 \times 10^{-3} \mathrm{~cm}$ & $2.02 \times 10^{-3} \mathrm{~cm}$ & $1.01 \times 10^{-3} \mathrm{~cm}$ & Cell size \\
\hline$\widetilde{\Delta x}^{-1}$ & 8 & 16 & 32 & $\delta_{L} / \Delta x$ \\
\hline$z_{T, 0}$ & & $1.95 \mathrm{~cm}=7.52 L$ & & Initial flame position along $z$-axis \\
\hline$\varepsilon$ & & $1.26 \times 10^{9} \mathrm{erg} / \mathrm{cm}^{3} \cdot \mathrm{s}$ & & Energy-injection rate \\
\hline$U_{\delta}$ & & $4.53 \times 10^{3} \mathrm{~cm} / \mathrm{s}=15 S_{L}$ & & Turbulent velocity at scale $\delta_{L}$ \\
\hline$U$ & & $9.07 \times 10^{3} \mathrm{~cm} / \mathrm{s}=30 S_{L}$ & & Turbulent velocity at scale $L$ \\
\hline$U_{r m s}$ & & $1.04 \times 10^{4} \mathrm{~cm} / \mathrm{s}=34.48 S_{L}$ & & Turbulent r.m.s. velocity \\
\hline$U_{l}$ & & $5.60 \times 10^{3} \mathrm{~cm} / \mathrm{s}=18.54 S_{L}$ & & Integral velocity \\
\hline$l$ & & $6.04 \times 10^{-2} \mathrm{~cm}=1.87 \delta_{L}$ & & Integral scale \\
\hline$\tau_{e d}$ & & $2.86 \times 10^{-5} \mathrm{~s}$ & & Eddy turnover time, $L / U$ \\
\hline$t_{i g n}$ & $3.0 \tau_{e d}$ & $3.0 \tau_{e d}$ & $2.0 \tau_{e d}$ & Time of ignition \\
\hline$t_{\text {total }}$ & & $16.0 \tau_{e d}$ & & Total simulation time \\
\hline$D a$ & & 0.05 & & Damköhler number, eq. (11) \\
\hline$L_{G}$ & & $9.47 \times 10^{-6} \mathrm{~cm}=2.96 \times 10^{-4} \delta_{L}$ & & Gibson scale, eq. (11) \\
\hline$M a_{F}$ & & 0.25 & & Mach number in fuel, $U / c_{s, F}$ \\
\hline$M a_{P}$ & & 0.09 & & Mach number in product, $U / c_{s, P}$ \\
\hline
\end{tabular}

\footnotetext{
${ }^{\text {a }}$ Parameters common to all simulations are shown only once in S2 column.
} 
Table 3. Time-Averaged Width and Speed of the Turbulent Flame Brush ${ }^{\mathrm{a}}$

\begin{tabular}{ccccc}
\hline \hline & $\overline{\delta_{T}} / \delta_{L}$ & $O\left(\overline{\delta_{T}} / \delta_{L}\right)$ & $\overline{S_{T}} / S_{L}$ & $O\left(\overline{S_{T}} / S_{L}\right)$ \\
\hline S1 & 16.13 & & 6.09 & \\
S2 & 14.86 & 1.96 & 4.50 & 2.29 \\
S3 & 14.42 & & 4.09 & \\
& & & &
\end{tabular}

a Time-averaging is performed over the time interval $(2-16) \tau_{e d}$. 


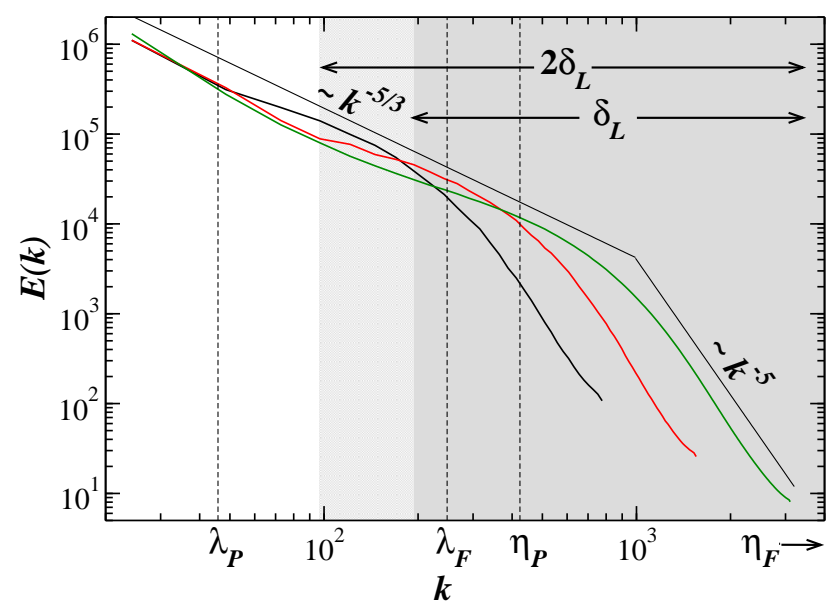

Figure 1 Instantaneous energy spectra for simulations S1 (black), S2 (red), and S3 (green) at a time immediately prior to ignition. Shaded regions illustrate scales associated with thermal width $\delta_{L}$ and full width $2 \delta_{L}$ of the laminar flame (cf. Fig.77). Vertical dashed lines show wavenumbers corresponding to the physical Taylor microscales in the product, $\lambda_{P}$, and fuel, $\lambda_{F}$, as well as the physical Kolmogorov scale in the product, $\eta_{P}$, based on the value of the viscosity coefficient discussed in $\$ 2.4$. The wavenumber corresponding to the Kolmogorov scale in the fuel, $\eta_{F}=1.18 \times 10^{-3}$ $\mathrm{cm}$, is located outside the range of the graph.
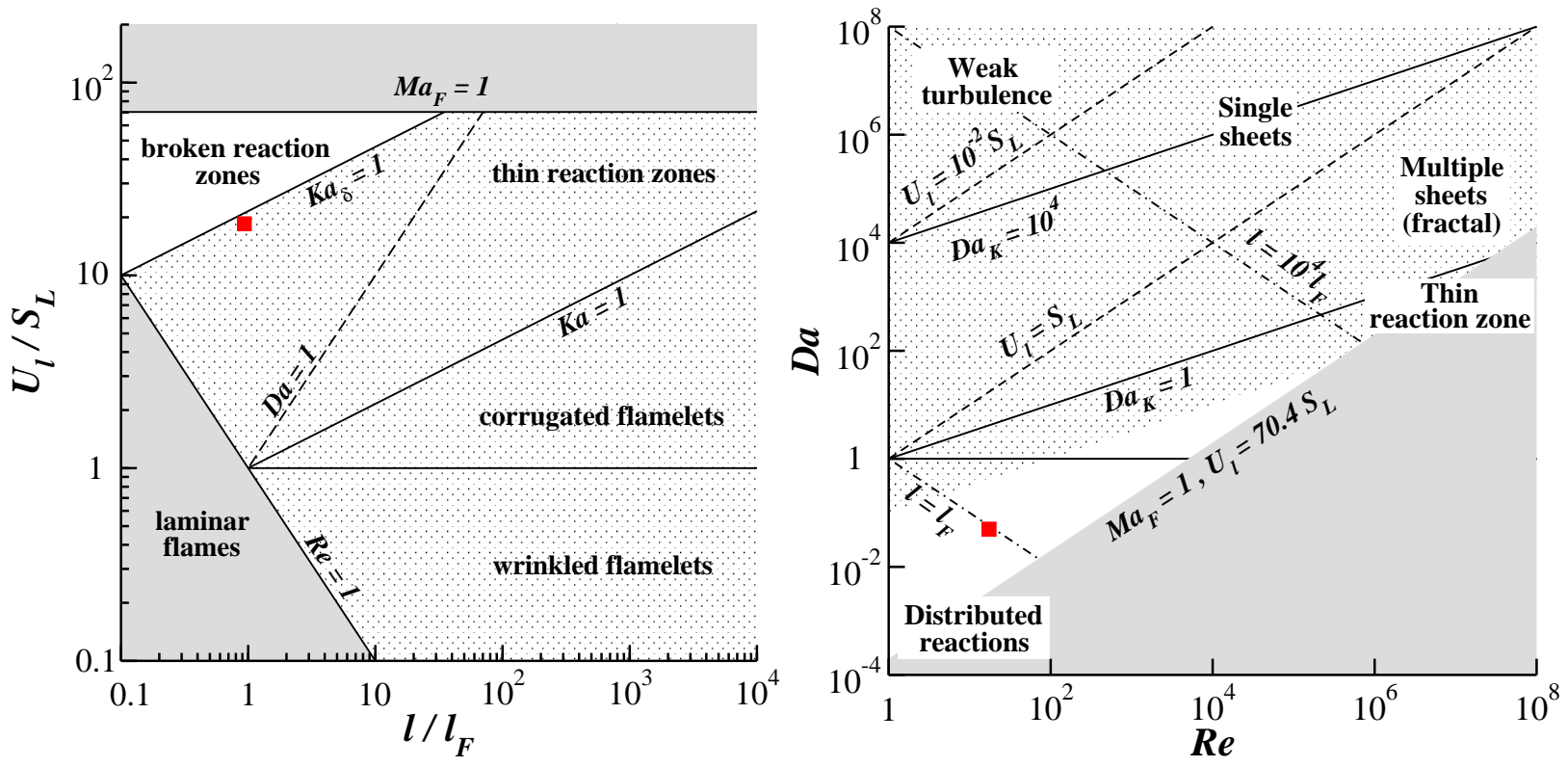

Figure 2 Combustion regime diagrams according to [1] (left) and [2] (right). Red square corresponds to the simulations presented in this work. Light shaded area shows the regime range in which the existence of flamelets is suggested. The traditional form of the diagrams was modified by adding the $M a_{F}=1$ line indicating the region of supersonic turbulence in the cold $\mathrm{H}_{2}$-air fuel under the atmospheric conditions. See text for further details. 

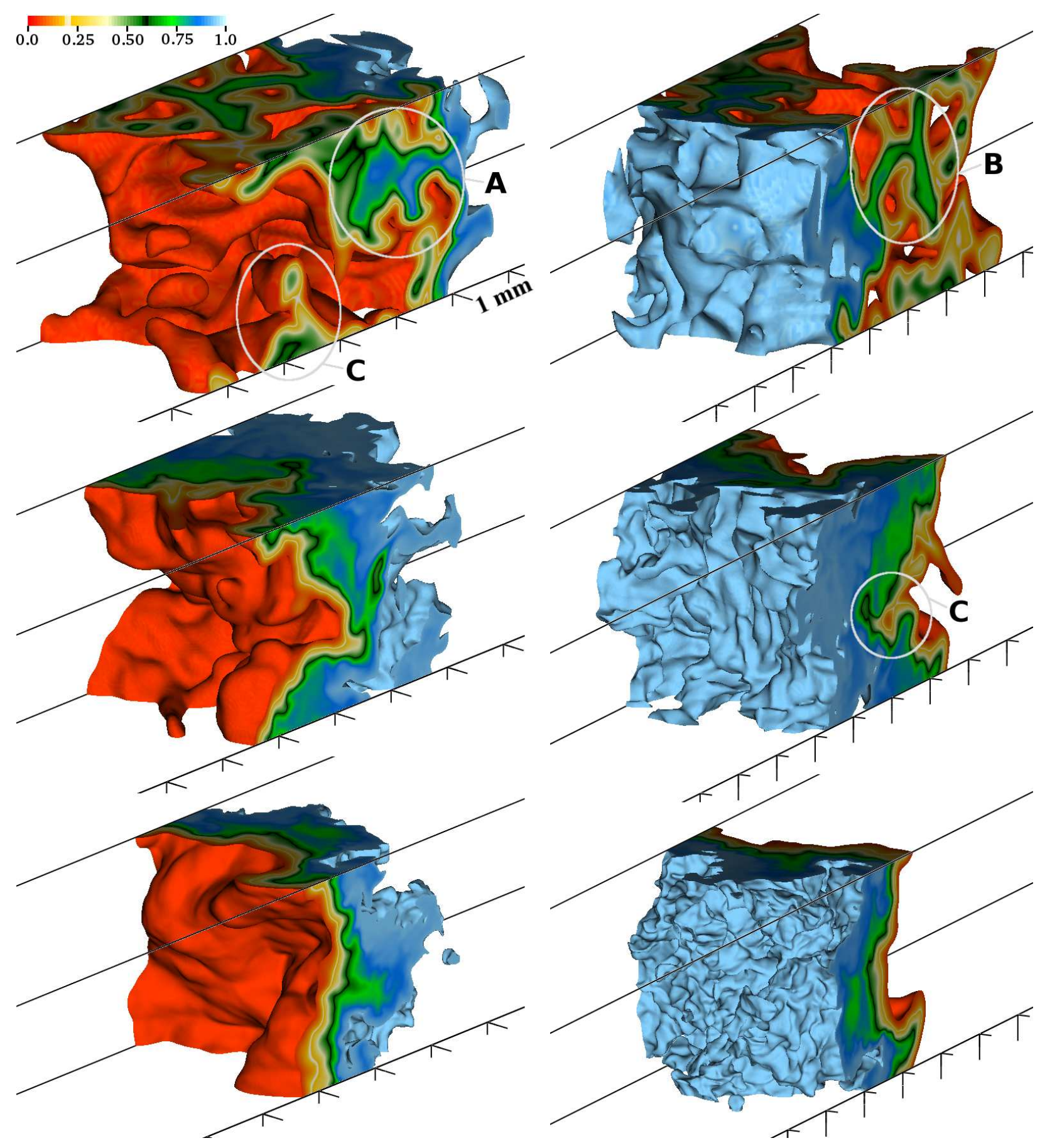

Figure 3 Flame brush structure in simulations S1 (top row), S2 (middle row), and S3 (bottom row). Shown is fuel mass fraction at $t=13 \tau_{e d}$. Left panels show view from the product side, right panels show view from the fuel side. Bounding isosurfaces represent $Y=0.05$ and $Y=0.95$. The thin black line, corresponding to $Y=0.6$, marks the boundary between the preheat and reaction zones. The thin white line, corresponding to $Y=0.2$, shows the location of the peak reaction rate (cf. Fig. 7). Regions A, B, and C are discussed in $\$ 3.1$ 

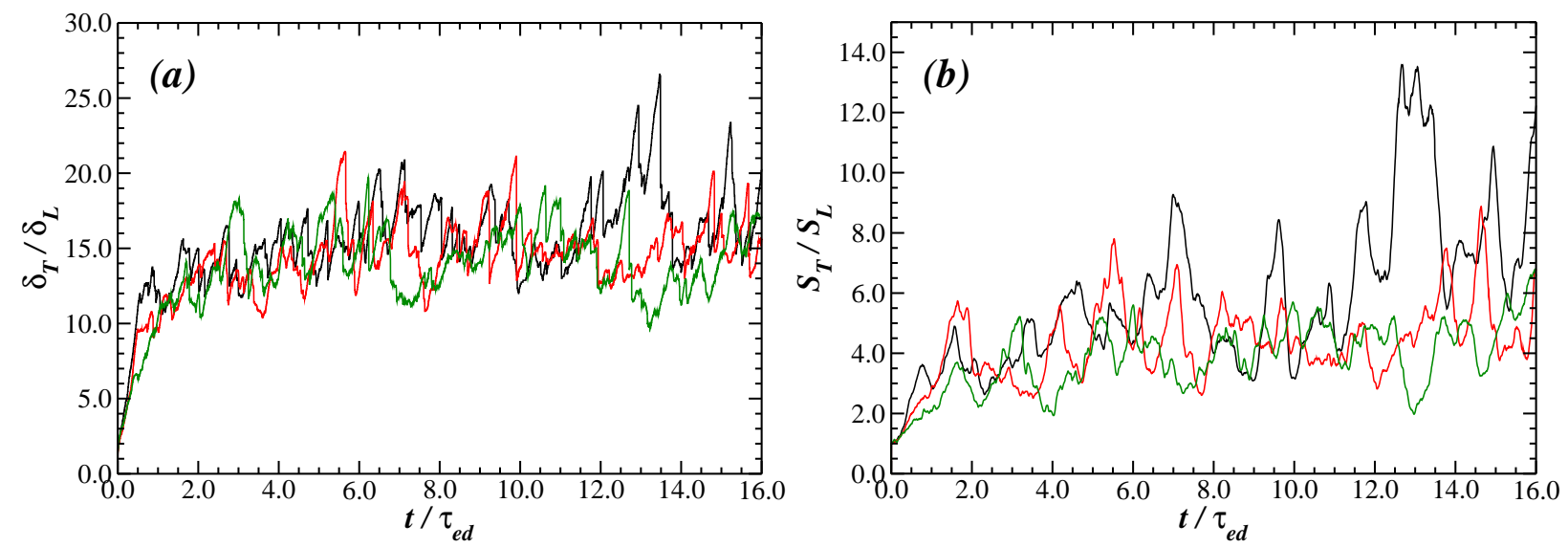

Figure 4 (a) Evolution of the turbulent flame width $\delta_{T}$ normalized by $\delta_{L}$. Note, that domain width $L=8 \delta_{L}$. (b) Evolution of the turbulent flame speed $S_{T}$ normalized by $S_{L}$. In both panels, black lines correspond to simulation $\mathrm{S} 1$, red to $\mathrm{S} 2$, and green to $\mathrm{S} 3$.

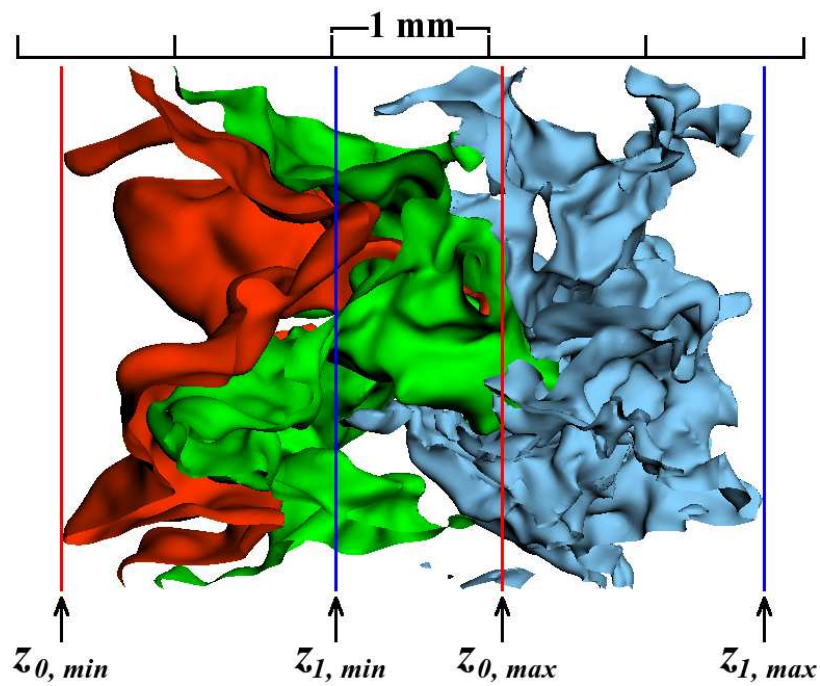

Figure 5 Isosurfaces of the fuel mass fraction in simulation S2 at $t=13 \tau_{\text {ed }}$ (cf. Fig. 3, middle row, left panel). Isosurface values are 0.05 (red), 0.6 (green), 0.95 (blue). Red and green isosurfaces bound the flamelet reaction zone. Green and blue isosurfaces bound the preheat zone. The $z_{0, \min }$ and $z_{1, \max }$ mark the flame-brush bounds. The $z_{0, \max }$ and $z_{1, \min }$ indicate, respectively, the maximum extents of product and fuel penetration into the flame brush. 


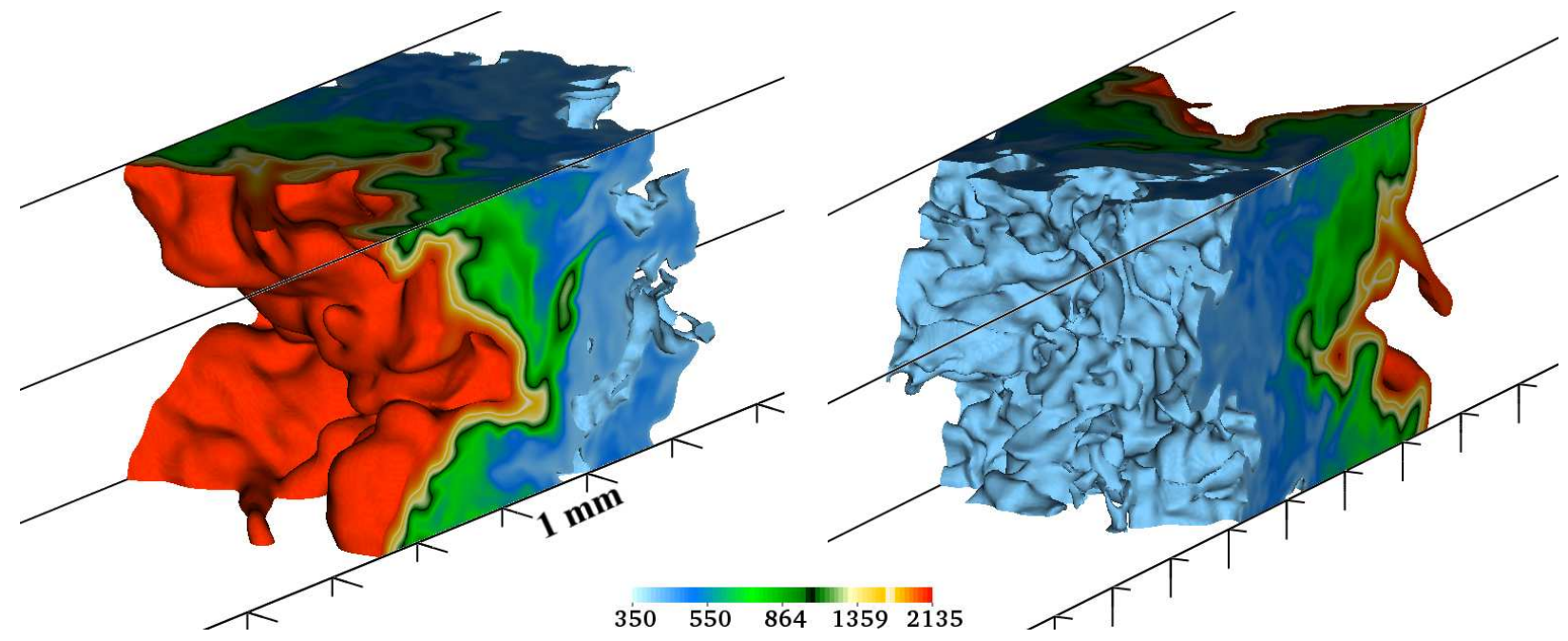

Figure 6 Temperature structure of the flame brush in simulation S2 at $t=13 \tau_{e d}$ (cf. Fig. 3, middle row). Left panel shows view from the product side, right panel show view from the fuel side. Bounding isosurfaces represent $T=400$ $\mathrm{K}$ and $T=2060 \mathrm{~K}$. Thin black line, corresponding to $T=1035 \mathrm{~K}$, marks the boundary between the preheat and reaction zones, while thin white line, corresponding to $T=1680 \mathrm{~K}$, shows the location of the peak reaction rate (cf. Fig. 7). The colormap is on a logarithmic scale. 


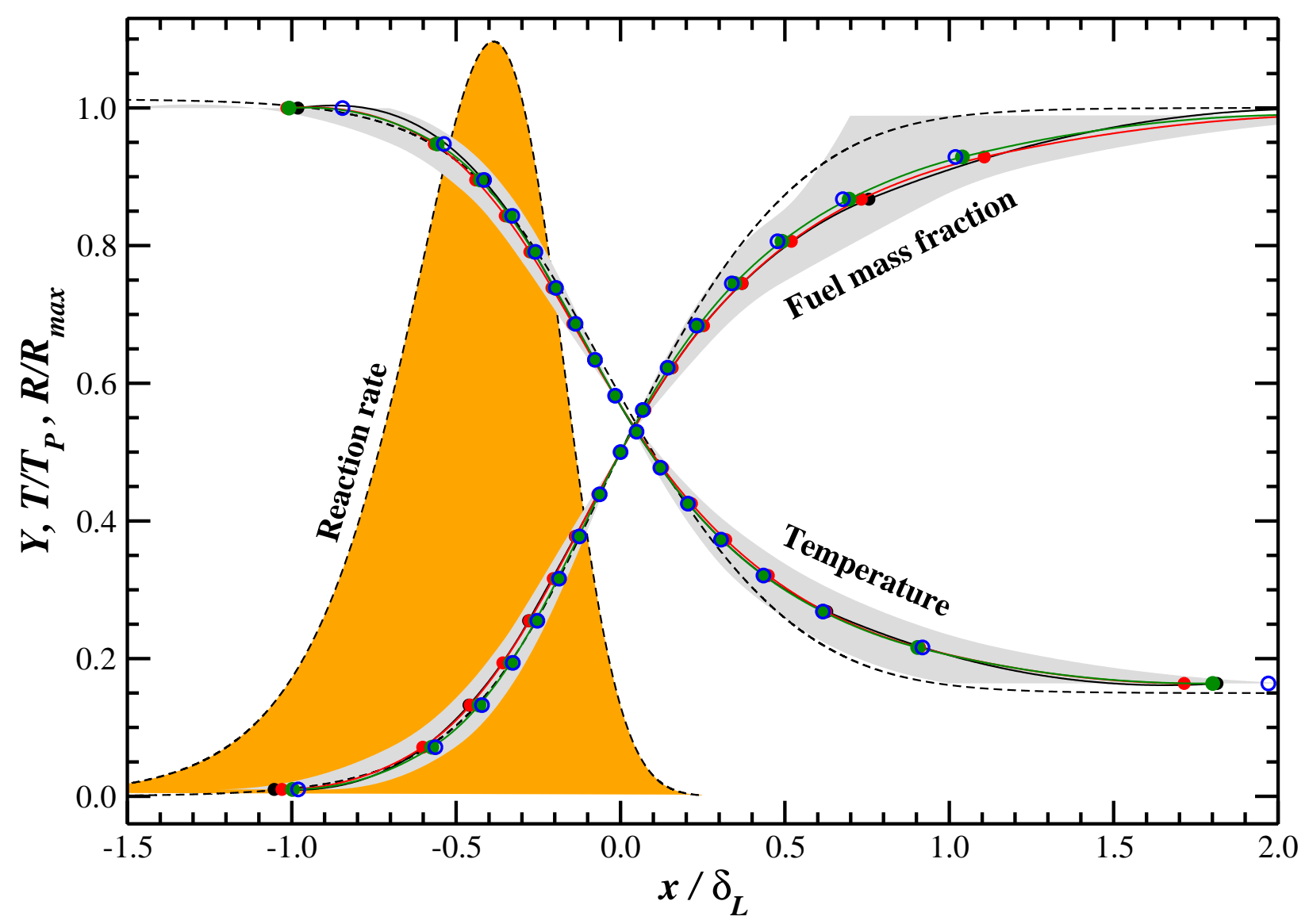

Figure 7 Time-averaged flamelet structure in the turbulent flame brush. Increasing curves represent fuel mass fraction, $Y$, decreasing curves represent temperature, $T$. Dashed lines are exact solutions for the planar laminar flame with the shaded orange region showing the reaction rate, $R$. Solid black lines correspond to simulation $\mathrm{S} 1$, red to $\mathrm{S} 2$, and green to $\mathrm{S} 3$. Circles represent calculated values and solid lines are the Akima spline fits. Time averaging is performed over the time interval $(2-16) \tau_{e d}$. Open blue circles represent flamelet structure obtained in S3 using half the time averaging interval, i.e., $(9-16) \tau_{e d}$. Shaded gray regions show the range of variability of individual profiles in S3 within the time-averaging interval with all instantaneous $Y$ profiles shifted to coincide at $Y(x=0)=0.5$ and $T$ profiles shifted to coincide at $\left(T(x=0)-T_{0}\right) /\left(T_{P}-T_{0}\right)=0.5$. The laminar flame structure is shown for the fuel temperature $320 \mathrm{~K}$ to account for the turbulent fuel heating in the simulation. $T$ and $R$ are normalized by their respective reference peak values in the laminar flame with the fuel temperature $T_{0}=293 \mathrm{~K}$, namely $T_{P}$ (see Table 1) and $R_{\max }=9.5 \times 10^{4} \mathrm{~s}^{-1}$, therefore maximum values of $T$ and $R$ in the profiles are larger than one. 

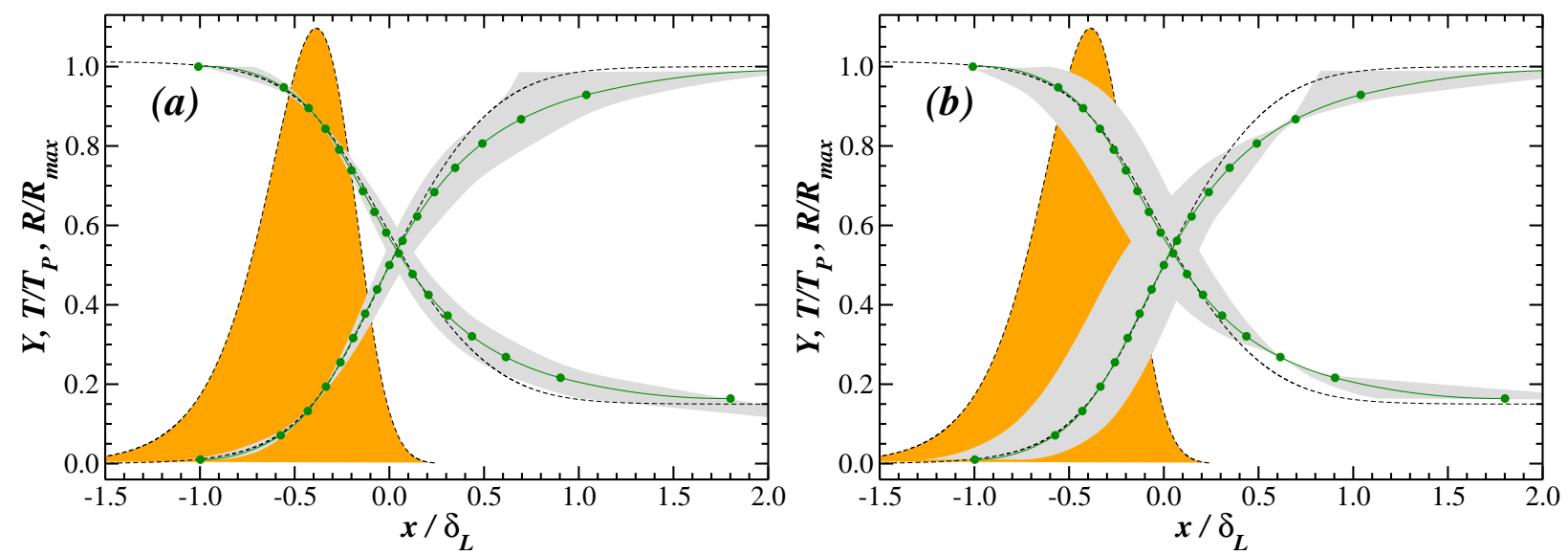

Figure 8 Variability range of instantaneous profiles of $Y$ and $T$ shifted to coincide, respectively, at $Y=0.15,(T-$ $\left.T_{0}\right) /\left(T_{P}-T_{0}\right)=0.85(\mathrm{a})$, and $Y=0.85,\left(T-T_{0}\right) /\left(T_{P}-T_{0}\right)=0.15(\mathrm{~b})$. Same as Fig. 7 with only data for simulation $\mathrm{S} 3$ shown. See text for further details.

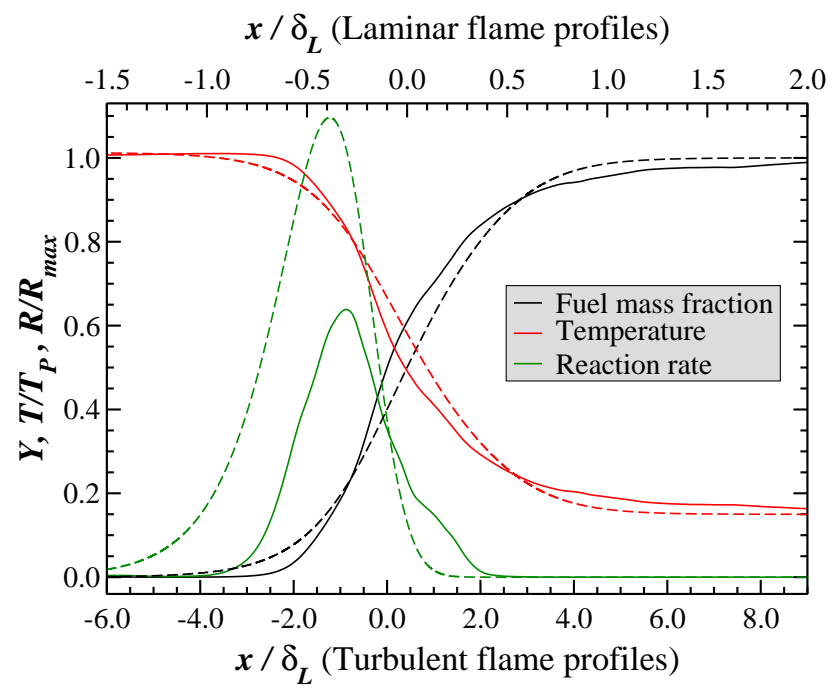

Figure 9 Instantaneous average distributions of the fuel mass fraction, $Y$, temperature, $T$, and the reaction rate, $R$, in the simulation S3 at time $t=12.8 \tau_{e d}$. Each profile represents the distribution of a given quantity along the $z$-axis, i.e., the longest dimension of the domain, with each point being the average over the $x-y$ plane. For comparison, corresponding profiles for the planar laminar flame are superimposed with dashed lines. The bottom coordinate scale is for the turbulent flame while the top scale is for the laminar flame. Note the difference in the range of these two scales with the turbulent flame profiles being more than a factor of 4 wider. As in Fig. 7 $T$ and $R$ are normalized by $T_{P}$ (see Table 1) and $R_{\max }=9.5 \times 10^{4} \mathrm{~s}^{-1}$, the laminar flame solution is shown for the fuel temperature $320 \mathrm{~K}$, and points of origin of the $x$-coordinates are chosen such that $Y(x=0)=0.5$ and $\left(T(x=0)-T_{0}\right) /\left(T_{P}-T_{0}\right)=0.5$. 


\section{Appendix A. Isosurface-based method for flamelet structure reconstruction}

Here we describe the properties of the method that we use to reconstruct the average flamelet structure in the turbulent flame brush, as discussed in $\$ 3.2$

First consider a planar laminar flame normal to $z$-axis. In this flame, all isosurfaces $\mathcal{S}_{i}$ are exactly parallel, $A_{i}=$ Const, and eq. (15) reduces to $\eta_{i+1} \equiv z\left(Y_{i+1}\right)-z\left(Y_{i}\right)$, where $z(Y)$ is based on the exact laminar flame profile $Y(z)$. Therefore, $Y(\eta)=Y(z)$. Here for brevity we only consider $Y$ and the same reasoning applies to $T$. Imagine now that we deform that planar flame without stretching it, and we ensure that at each point curvature radius is much larger than the flame width. In this case, all isosurfaces remain exactly parallel, $A_{i}$ remains constant, and again, regardless of how complex the deformation is, this method will recover the exact laminar flame profile $Y(\eta)=Y(z)$.

Next consider a real flame subject to the action of turbulence. In this case, there is no reason to assume a priori either that isosurfaces $\mathcal{S}_{i}$ and $\mathcal{S}_{i+1}$ are parallel or that their surface areas are equal. Let us first define the distance $d\left(p, \mathcal{S}_{i+1}\right)$ between a point $p$, located on isosurface $\mathcal{S}_{i}$, and isosurface $\mathcal{S}_{i+1}$ as

$$
d\left(p, \mathcal{S}_{i+1}\right)=\min _{p^{\prime} \in \mathcal{S}_{i+1}}\left\|p-p^{\prime}\right\|_{2},
$$

where $\|.\|_{2}$ is the Euclidean norm. Then we define the maximum distance $\xi_{\max }$ between the isosurfaces $\mathcal{S}_{i}$ and $\mathcal{S}_{i+1}$ in a traditional way as the Hausdorff distance

$$
\xi_{\text {max }} \equiv d_{\text {max }}\left(\mathcal{S}_{i}, \mathcal{S}_{i+1}\right)=\max \left\{\max _{p \in \mathcal{S}_{i}}\left(d\left(p, \mathcal{S}_{i+1}\right)\right), \max _{p^{\prime} \in \mathcal{S}_{i+1}}\left(d\left(p^{\prime}, \mathcal{S}_{i}\right)\right)\right\} .
$$

Similarly we define the minimum distance $\xi_{\min }$ between $\mathcal{S}_{i}$ and $\mathcal{S}_{i+1}$

$$
\xi_{\text {min }} \equiv d_{\text {min }}\left(\mathcal{S}_{i}, \mathcal{S}_{i+1}\right)=\min \left\{\min _{p \in \mathcal{S}_{i}}\left(d\left(p, \mathcal{S}_{i+1}\right)\right), \min _{p^{\prime} \in \mathcal{S}_{i+1}}\left(d\left(p^{\prime}, \mathcal{S}_{i}\right)\right)\right\} .
$$

Using these definitions, the following proposition can be made:

As $n \rightarrow \infty$ and $\Delta Y \equiv Y_{i+1}-Y_{i} \rightarrow 0$, then $\mathcal{S}_{i+1} \rightarrow \mathcal{S}_{i}$ in the sense that as $\xi_{\min } \rightarrow 0, \xi_{\max } \rightarrow \xi_{\text {min }}$.

A corollary of the above proposition is the statement that $A_{i} \rightarrow A_{i+1}$ as $Y_{i} \rightarrow Y_{i+1}$.

In order to prove this proposition, we first make several observations. Isosurfaces cannot intersect each other, since that would lead to infinite gradients and unphysical conditions. Second, since the evolution of $Y$ is governed partly by diffusion and conduction, and there are no shocks in the system, the distribution of $Y$ is smooth and continuous. Moreover, $Y$ cannot be exactly constant over any extended region since advective and diffusive processes in an unsteady turbulent flow would inevitably create spatial variations in the distribution of $Y$ on all scales. Therefore, isosurfaces corresponding to all values $Y_{i}<Y<Y_{i+1}$ are all bounded by the isosurfaces $\mathcal{S}_{i}$ and $\mathcal{S}_{i+1}$, they are smoothly distributed throughout the volume bounded by $\mathcal{S}_{i}$ and $\mathcal{S}_{i+1}$, and as $Y_{i} \rightarrow Y_{i+1}, \xi_{\min } \rightarrow 0$.

Let us first prove that a value $Y^{\prime} \in\left(Y_{i}, Y_{i+1}\right)$ can always be found such that for its isosurface $\mathcal{S}^{\prime}$ the following is true: $\xi_{\min }-\xi_{\min }^{\prime} \leq \xi_{\max }-\xi_{\max }^{\prime}$. Here $\xi_{\max }^{\prime} \equiv d_{\max }\left(\mathcal{S}^{\prime}, \mathcal{S}_{i+1}\right)$ and $\xi_{\min }^{\prime} \equiv d_{\min }\left(\mathcal{S}^{\prime}, \mathcal{S}_{i+1}\right)$ by analogy with eqs. (A.2)-(A.3). Assume the contrary, namely that for all values of $Y^{\prime}$ from the interval $\left(Y_{i}, Y_{i+1}\right)$ we have $\xi_{\min }-\xi_{\min }^{\prime}>\xi_{\max }-\xi_{\max }^{\prime}$. This means that as $Y^{\prime} \rightarrow Y_{i+1}, \xi_{\min }^{\prime} \rightarrow 0$ and $\xi_{\max }^{\prime}>\xi_{\max }-\xi_{\min }$. Therefore, $\mathcal{S}^{\prime}$ isosurfaces have some limiting isosurface $\mathcal{S}^{*}$ which has the same value of $Y^{*}=Y_{i+1}$ but which is distinct from $\mathcal{S}_{i+1}$ because $\xi_{\max }^{*}>\xi_{\max }-\xi_{\min }$. Since isosurfaces do not intersect, this means that no other isosurfaces pass through the volume bounded by $\mathcal{S}^{*}$ and $\mathcal{S}_{i+1}$. Consequently, $\mathcal{S}^{*}$ and $\mathcal{S}_{i+1}$ bound a region of constant $Y$. We discussed above, however, that such regions cannot exist. Therefore, we arrive at a contradiction and our assumption was wrong, thus proving our initial statement.

It then follows, that there exists a value $Y^{\prime \prime} \in\left(Y^{\prime}, Y_{i+1}\right)$ such that for its isosurface $\mathcal{S}^{\prime \prime}$ we have $\xi_{\min }^{\prime}-\xi_{\min }^{\prime \prime} \leq$ $\xi_{\max }^{\prime}-\xi_{\max }^{\prime \prime}$. By induction this proves the original proposition. A similar result can be proven for temperature.

In practice, this result means the following. The value of $\xi_{\max }-\xi_{\min }$ is the measure of the variation of $\mathcal{S}_{i}$ with respect to $\mathcal{S}_{i+1}$. As we select finer discretization intervals of $Y$, this variation decreases while $\xi_{\min } \rightarrow 0$. Therefore, the isosurfaces become more and more parallel to each other. An illustration of this can be seen in Fig. 5 While there is very little in common between the $Y=0.05$ and $Y=0.95$ isosurfaces, $Y=0.6$ tends to follow the $Y=0.05$ isosurface much more closely.

An important question concerns the choice of the number of discretization intervals $n$ in practical applications. It 
follows from the above discussion, that in order to maximize the accuracy of the method, $n$ must be chosen as large as possible. This ensures that consecutive isosurfaces are close to each other and that $\xi_{\max }-\xi_{\min }$ is minimal or, ideally, zero. In the computational domain, the minimum spatial scale is set by the cell size $\Delta x$. This determines the minimum practical separation of isosurfaces $\xi_{\min }$. Isosurfaces with smaller separations would pass through the same cell, which would result in a substantial drop in accuracy of the overall method since all flow variables are piecewise constant within a cell. At the same time, if two isosurfaces on average have separation of a few $\Delta x$, this ensures that their surface areas are close in value, and there cannot exist an intermediate isosurface which is substantially more or less tightly folded. These considerations allow us to determine the maximum value of $n$.

If we assume that the maximum gradient in temperature in the domain is close to that in the laminar flame profile $(d T / d x)_{L, \max }$, then

$$
\frac{\Delta T_{\min }}{(d T / d x)_{L, \max }}=\xi_{\min } \approx \Delta x \text {. }
$$

Recalling that $\delta_{L}=\left(T_{P}-T_{0}\right) /(d T / d x)_{L, \max }$, we can rewrite eq. (A.4) as

$$
\frac{T_{\max }-T_{\min }}{n}=\frac{T_{P}-T_{0}}{\delta_{L}} \Delta x,
$$

where $T_{\min }$ and $T_{\max }$ are the lower and upper bounds of the discretized temperature range. Since $T_{\min } \approx T_{0}$ and $T_{\max }=T_{P}$, we find that

$$
n_{\max } \sim \frac{\delta_{L}}{\Delta x}
$$

A similar argument applies to the fuel mass fraction. Therefore, $n$ should not be larger than the number of grid cells within the laminar flame thermal width. Typically, we find the choice of $n$ based on eq. A.6) adequate in practical applications.

We found that adaptivity of the algorithm, which implements the method described here, is essential to guarantee high accuracy. The estimate given by eq. (A.6) assumed that the largest gradients present in the system are well approximated by $(d Y / d x)_{L, \max }$ and $(d T / d x)_{L, \max }$ in the laminar flame profile. This may not be the case. Therefore, it is important for the algorithm to be able to decrease $n$ if larger gradients are encountered. On the other hand, the choice of $n$ in eq. A.6 is based on the thermal flame width. The full flame width is larger than $\delta_{L}$. Therefore, while eq. A.6) is adequate to capture flame structure in the steepest region, the parts of the profile close to the extreme values of the discretized range may be under-resolved (see Fig. 7). In those regions, both $Y$ and $T$ vary slowly in space. Thus, for consecutive isosurfaces, $\eta_{i} \gg \Delta x$. Ideally, $\Delta Y$ and $\Delta T$ must be chosen such that $\Delta x \lesssim \eta_{i} \lesssim \alpha \Delta x$ for $i \in[1, n]$. Typically, we find that $\alpha=4$ is a reasonable choice.

Then the adaptivity of the algorithm is implemented as follows. The value of $n$ is adjusted to ensure that both $\Delta Y$ and $\Delta T$ are marginally large enough to prevent situations when $\eta_{i}<\Delta x$. On the other hand, if $\eta_{i}>\alpha \Delta x$ is encountered, $\Delta Y$ or $\Delta T$ for that interval is divided by two. Subsequently, $\eta$ is evaluated separately for the first and second half-intervals, and the final $\eta_{i}$ is the sum of those values. If any of the intermediate values of $\eta$ is larger than $\alpha \Delta x$, that half-interval is again subdivided by two and the whole procedure proceeds recursively until on each of the substeps the condition $\eta<\alpha \Delta x$ is satisfied. This ensures that $\eta$ is always determined for isosurfaces which are close to each other.

Note, that this algorithm uses the laminar flame structure only to provide the initial guess for $n$. Subsequently, it adapts the discretization intervals for $Y$ and $T$ based only on the actual gradients found in the flow. Therefore, information about the laminar flame structure is not required at all, and eq. A.6 only facilitates finding the correct $\Delta Y$ and $\Delta T$. At the same time, in situations when the internal structure of the turbulent flame is very different from that of the laminar flame, as would be expected in the case of distributed burning, eq. A.6 is only of limited benefit.

A key limitation of this method is that it can be applied only to quantities that change monotonically through the flame, such as $Y, T$, or $\rho$. In particular, this procedure cannot be used to directly determine the distribution of the reaction rate, $R$. Non-monotonic behavior of a quantity means that there is an inherent degeneracy in its distribution inside the flame. Consider a planar laminar flame (e.g., see Fig. 7). Two distinct points in the reaction rate profile have the same value of $R$. Therefore, there would be two isosurfaces for each value of $R_{i}$ and $R_{i+1}$. Volume $V_{i}$ would be the total volume bounded by both pairs of isosurfaces and area $A_{i}$ would be the combined surface area of both isosurfaces $\mathcal{S}_{i}^{\prime}$ and $\mathcal{S}_{i}^{\prime \prime}$. There is no mechanism in this method to distinguish the contributions of each pair of isosurfaces both into 
$A_{i}$ and $V_{i}$. Consequently, $\eta_{i}$ found using eq. (15) would have no meaning.

In general, we find that the method described above performs best for quantities that predominantly change within the volume of the flame brush, and that do not vary at all, or vary only slightly around the limiting values of the discretized range, outside the flame brush. This is the case for $Y$ and $T$. The accuracy is the highest for $Y$ which is exactly 0 or 1 outside the flame brush. Therefore, results are not contaminated by the isosurfaces which pass outside the burning region. For temperature, the accuracy is marginally lower due to the fluctuations in the turbulent field away from the flame brush, which can cause values of $T$ to become slightly below the upper bound or above the lower bound. As a result, isosurfaces would pass through such regions which are not part of the flame brush. Typically, however, this can introduce small errors only near the extreme values in the temperature profile. At the same time, for a quantity like density, the accuracy drops to the point that the obtained profiles cannot be reliably used to analyze the density structure of the flamelets. This is primarily due to the fact that variations in density outside the flame brush are large enough to be significant in comparison with the change in $\rho$ inside the flame brush itself. Therefore, a large portion of the profile ends up being contaminated by the contributions from the regions which are outside the flame brush, but which happen to have values of $\rho$ that fall within its discretized range.

Finally, this method does not provide any means to relate the distributions of different quantities, e.g., $Y$ and $T$. In a planar laminar-flame structure, for instance, a given value of $Y$ uniquely defines its position $z$ in the profile, and thus it uniquely determines the value of $T=T(z)$. This cannot be done for the profiles obtained using the method described above. Recall that $\eta$ is not a spatial coordinate but simply the distance between consecutive isosurface values, and it is assumed that $\eta_{0}=0$. Thus, values of $\eta$ for $Y$ and $T$ are distinct and unrelated and other arguments must be invoked in order to relate their distributions.

\section{References}

[1] N. Peters, Turbulent Combustion, Cambridge University Press (2000)

[2] P.A. Libby, F.A. Williams, in Turbulent Reacting Flows, eds. P.A. Libby, F.A. Williams, Academic Press (1994) 1-61

[3] F.A. Williams, in Smart Control of Turbulent Combustion, ed. A. Yoshida, Springer-Verlag (2001) 1-11

[4] G. Damköhler, Z. Elektrochem. und Angewandte Physikalische Chemie 46 (1940) 601

[5] J.F. Driscoll, Prog. Ener. Comb. Sci. 34 (2008) 91-134

[6] R.W. Bilger, S.B. Pope, K.N.C. Bray, J.F. Driscoll, Proc. Combustion Inst. 30 (2005) 21-42

[7] A. Buschmann, F. Dinkelacker, T. Schafer, M. Schafer, J. Wolfrum, Proc. Combustion Inst. 26 (1996) 437-445

[8] F. Dinkelacker, A. Soika, D. Most, D. Hofmann, A. Leipertz, W. Polifke, et al., Proc. Combustion Inst. 27 (1998) $857-865$

[9] M.S. Mansour, N. Peters, Y.-C. Chen, Proc. Combustion Inst. 27 (1998) 767-773

[10] M.J. Dunn, A.R. Masri, R.W. Bilger, Comb. Flame 151 (2007) 46-60

[11] M. Boger, D. Veynante, H. Boughanem, A. Trouvé, 27th Symp. (Int.) on Combustion, The Combustion Institute, Pittsburgh, (1998) 917-925

[12] J.B.Bell, M.S.Day, J.F.Grcar, Proc. Combustion Inst. 29 (2002) 1987-1993

[13] M. Tanahashi, Y. Nada, Y. Ito, T. Miyauchi, Proc. Combustion Inst. 29 (2002) 2041-2049

[14] R. Sankaran, E.R. Hawkes, J.H. Chen, Proc. Combustion Inst. 31 (2006) 1291-1298

[15] A.J. Aspden, J.B. Bell, M.S. Day, S.E. Woosley, M. Zingale, Astrophys. J. 689 (2008) 1173-1185

[16] A. Kolmogorov, Dokl. Akad. Nauk SSSR 31 (1941) 538-541

[17] V.N. Gamezo, T. Ogawa, E.S. Oran, Comb. Flame 155 (2008) 302-315

[18] V.N. Gamezo, T. Ogawa, E.S. Oran, AIAA-2009-440 (2009)

[19] A.Y. Poludnenko, T.A. Gardiner, E.S. Oran, J. Fluid Mech. (in preparation)

[20] J.M. Stone, T.A. Gardiner, P. Teuben, J.F. Hawley, J.B. Simon, Astrophys. J. Supp. 178 (2008) 137-177

[21] T.A. Gardiner, J.M. Stone, J. Comp. Phys. 227 (2008) 4123-4141

[22] J.M. Stone, T. Gardiner, New Astronomy 14 (2009) 139-148

[23] P. Colella, J. Comp. Phys. 87 (1990) 171-200

[24] J. Saltzman, J. Comp. Phys. 115 (1994) 153-168

[25] P. Colella, P.R. Woodward, J. Comp. Phys. 54 (1984) 174-201

[26] J.M. Stone, E.C. Ostriker, C.F. Gammie, Astrophys. J. 508 (1998) L99-L102

[27] M.N. Lemaster, J.M. Stone, Astrophys. J. 691 (2009) 1092-1108

[28] J.P. Boris, in Whither Turbulence? Turbulence at the Crossroads, ed. J.L. Lumley, Springer-Verlag (1990) 344-353

[29] D.H. Porter, A. Pouquet, P.R. Woodward, Theor. Comput. Fluid Dynamics 4 (1992) 13-49

[30] J. Liu, E.S. Oran, C.R. Kaplan, J. Comp. Phys. 208 (2005) 416-434

[31] F.F. Grinstein, L.G. Margolin, W.J. Rider, Implicit Large Eddy Simulation, Cambridge Univ. Press (2007)

[32] Ya.B. Zel'dovich, J. Appl. Mech. \& Tech. Phys. 7 (1966) 68-69

[33] Ya.B. Zel'dovich, A.G. Istratov, N.I. Kidin, V.B. Librovich, Combustion Sci. Tech. 24 (1980) 1-13

[34] C. Meneveau, T. Poinsot, Comb. Flame 86 (1991) 311-332

[35] A.M. Khokhlov, Astrophys. J. 449 (1995) 695-713

[36] P.A. Davidson, Turbulence. An Introduction for Scientists and Engineers, Oxford University Press (2004) 
[37] A.Y. Poludnenko, E.S. Oran, Comb. Flame (in preparation) 